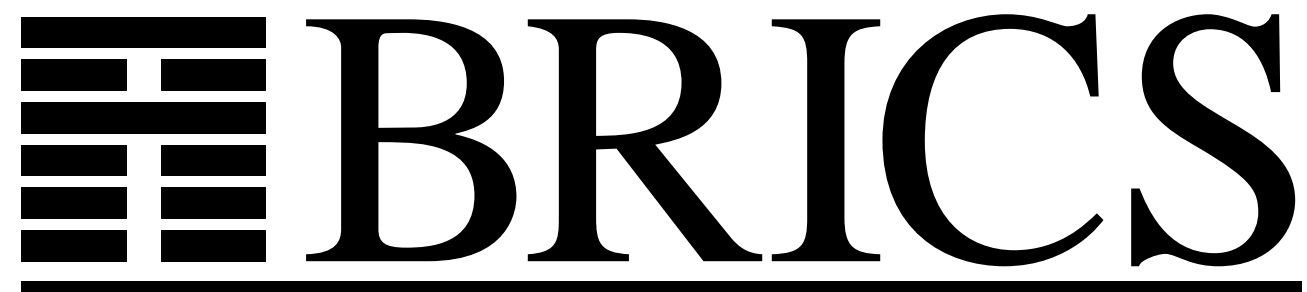

Basic Research in Computer Science

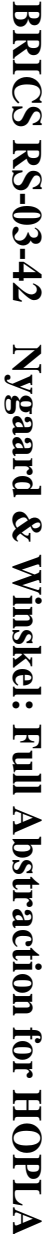

\title{
Full Abstraction for HOPLA
}

Mikkel Nygaard

Glynn Winskel 
Copyright (c) 2003, Mikkel Nygaard \& Glynn Winskel.

BRICS, Department of Computer Science

University of Aarhus. All rights reserved.

Reproduction of all or part of this work is permitted for educational or research use on condition that this copyright notice is included in any copy.

See back inner page for a list of recent BRICS Report Series publications. Copies may be obtained by contacting:

\author{
BRICS \\ Department of Computer Science \\ University of Aarhus \\ Ny Munkegade, building 540 \\ DK-8000 Aarhus C \\ Denmark \\ Telephone: +4589423360 \\ Telefax: $\quad+4589423255$ \\ Internet: BRICS@brics.dk
}

BRICS publications are in general accessible through the World Wide Web and anonymous FTP through these URLs:

http://www.brics.dk

ftp: / / ftp.brics.dk

This document in subdirectory RS / $03 / 42$ / 


\title{
Full Abstraction for HOPLA
}

\author{
Mikkel Nygaard \\ BRICS* \\ University of Aarhus
}

\author{
Glynn Winskel \\ Computer Laboratory \\ University of Cambridge
}

\begin{abstract}
A fully abstract denotational semantics for the higher-order process language HOPLA is presented. It characterises contextual and logical equivalence, the latter linking up with simulation. The semantics is a clean, domain-theoretic description of processes as downwardsclosed sets of computation paths: the operations of HOPLA arise as syntactic encodings of canonical constructions on such sets; full abstraction is a direct consequence of expressiveness with respect to computation paths; and simple proofs of soundness and adequacy shows correspondence between the denotational and operational semantics.
\end{abstract}

\section{Introduction}

HOPLA (Higher-Order Process LAnguage [19]) is an expressive language for higher-order nondeterministic processes. It has a straightforward operational semantics supporting a standard bisimulation congruence, and can directly encode calculi like CCS, higher-order CCS and mobile ambients with public names. The language came out of work on a linear domain theory for concurrency, based on a categorical model of linear logic and associated comonads $[4,18]$, the comonad used for HOPLA being an exponential ! of linear logic.

The denotational semantics given in [19] interpreted processes as presheaves. Here we consider a "path semantics" for HOPLA which allows us to characterise operationally the distinguishing power of the notion of computation path underlying the presheaf semantics (in contrast to the distinguishing power of the presheaf structure itself). Path semantics is similar to trace

\footnotetext{
*Basic Research in Computer Science (www.brics.dk), funded by the Danish National Research Foundation.
} 
semantics [10] in that processes denote downwards-closed sets of computation paths and the corresponding notion of process equivalence, called path equivalence, is given by equality of such sets; computation paths, however, may have more structure than traditional traces. Indeed, we characterise contextual equivalence for HOPLA as path equivalence and show that this coincides with logical equivalence for a fragment of Hennessy-Milner logic which is characteristic for simulation equivalence in the case of image-finite processes [8].

To increase the expressiveness of HOPLA (for example, to include the type used in [25] for CCS with late value-passing), while still ensuring that every operation in the language has a canonical semantics, we decompose the "prefix-sum" type $\Sigma_{\alpha \in A} \alpha \cdot \mathbb{P}_{\alpha}$ in [19] into a sum type $\Sigma_{\alpha \in A} \mathbb{P}_{\alpha}$ and an anonymous action prefix type $! \mathbb{P}$. The sum type, also a product, is associated with injection ("tagging") and projection term constructors, $\beta t$ and $\pi_{\beta} t$ for $\beta \in A$. The prefix type is associated with constructions of prefixing ! $t$ and prefix match $[u>! x \Rightarrow t]$, subsuming the original terms $\beta . t$ and $[u>\beta . x \Rightarrow$ $t]$ using $\beta ! t$ and $\left[\pi_{\beta} u>! x \Rightarrow t\right]$.

In Sect. 2 we present a domain theory of path sets, used in Sect. 3 to give a fully abstract denotational semantics to HOPLA. Section 4 presents the operational semantics of HOPLA, essentially that of [19], and relates the denotational and operational semantics with pleasingly simple proofs of soundness and adequacy. Section 5 concludes with a discussion of related and future work.

\section{Domain Theory from Path Sets}

In the path semantics, processes are represented as collections of computation paths. Paths are elements of preorders $\mathbb{P}, \mathbb{Q}, \ldots$ called path orders which function as process types, each describing the set of possible paths for processes of that type together with their sub-path ordering. A process of type $\mathbb{P}$ is then represented as a downwards-closed subset $X \subseteq \mathbb{P}$, called a path set. Path sets $X \subseteq \mathbb{P}$ ordered by inclusion form the elements of the poset $\widehat{\mathbb{P}}$ which we'll think of as a domain of meanings of processes of type $\mathbb{P}$.

The poset $\widehat{\mathbb{P}}$ has many interesting properties. First of all, it is a complete lattice with joins given by union. In the sense of Hennessy and Plotkin [7], $\widehat{\mathbb{P}}$ is a "nondeterministic domain", with joins used to interpret nondeterministic sums of processes. Accordingly, given a family $\left(X_{i}\right)_{i \in I}$ of elements of $\widehat{\mathbb{P}}$, we sometimes write $\Sigma_{i \in I} X_{i}$ for their join. A typical finite join is written $X_{1}+\cdots+X_{k}$ while the empty join is the empty path set, the inactive process, written $\varnothing$. 
A second important property of $\widehat{\mathbb{P}}$ is that any $X \in \widehat{\mathbb{P}}$ is the join of certain "prime" elements below it; $\widehat{\mathbb{P}}$ is a prime algebraic complete lattice [17]. Primes are down-closures $\mathrm{y}_{\mathbb{P}} p=\left\{p^{\prime}: p^{\prime} \leq_{\mathbb{P}} p\right\}$ of individual elements $p \in \mathbb{P}$, representing a process that may perform the computation path $p$. The map $\mathrm{y}_{\mathbb{P}}$ reflects as well as preserves order, so that $p \leq_{\mathbb{P}} p^{\prime}$ iff $\mathrm{y}_{\mathbb{P}} p \subseteq \mathrm{y}_{\mathbb{P}} p^{\prime}$, and $\mathrm{y}_{\mathbb{P}}$ thus "embeds" $\mathbb{P}$ in $\widehat{\mathbb{P}}$. We clearly have $\mathrm{y}_{\mathbb{P}} p \subseteq X$ iff $p \in X$ and prime algebraicity of $\widehat{\mathbb{P}}$ amounts to saying that any $X \in \widehat{\mathbb{P}}$ is the union of its elements:

$$
X=\bigcup_{p \in X} \mathrm{y}_{\mathbb{P}} p
$$

Finally, $\widehat{\mathbb{P}}$ is characterised abstractly as the free join-completion of $\mathbb{P}$, meaning (i) it is join-complete and (ii) given any join-complete poset $C$ and a monotone map $f: \mathbb{P} \rightarrow C$, there is a unique join-preserving map $f^{\dagger}: \widehat{\mathbb{P}} \rightarrow C$ such that the diagram on the left below commutes.

$$
\mathbb{P} \underset{f}{\stackrel{\mathrm{y} \mathbb{P}}{\longrightarrow} \underset{C}{\mathbb{P}}} \mathfrak{d}^{f^{\dagger}} \quad f^{\dagger} X=\bigcup_{p \in X} f p
$$

We call $f^{\dagger}$ the extension of $f$ along $\mathrm{y}_{\mathbb{P}}$. Uniqueness of $f^{\dagger}$ follows from (1).

Notice that we may instantiate $C$ to any poset of the form $\widehat{\mathbb{Q}}$, drawing our attention to join-preserving maps $\widehat{\mathbb{P}} \rightarrow \widehat{\mathbb{Q}}$. By the freeness property $(2)$, join-preserving maps $\widehat{\mathbb{P}} \rightarrow \widehat{\mathbb{Q}}$ are in bijective correspondence with monotone maps $\mathbb{P} \rightarrow \widehat{\mathbb{Q}}$. Each element $Y$ of $\widehat{\mathbb{Q}}$ can be represented using its "characteristic function", a monotone map $f_{Y}: \mathbb{Q}^{\text {op }} \rightarrow \mathbf{2}$ from the opposite order to the simple poset $0<1$ such that $Y=\left\{q: f_{Y} q=1\right\}$ and $\widehat{\mathbb{Q}} \cong\left[\mathbb{Q}^{\text {op }}, \mathbf{2}\right]$. Uncurrying then yields the following chain:

$$
[\mathbb{P}, \widehat{\mathbb{Q}}] \cong\left[\mathbb{P},\left[\mathbb{Q}^{\mathrm{op}}, \mathbf{2}\right]\right] \cong\left[\mathbb{P} \times \mathbb{Q}^{\mathrm{op}}, \mathbf{2}\right]=\left[\left(\mathbb{P}^{\mathrm{op}} \times \mathbb{Q}\right)^{\mathrm{op}}, \mathbf{2}\right] \cong \widehat{\mathbb{P o p} \times \mathbb{Q}}
$$

So the order $\mathbb{P}^{\text {op }} \times \mathbb{Q}$ provides a function space type. We'll now investigate what additional type structure is at hand.

\subsection{Linear and Continuous Categories}

Write Lin for the category with path orders $\mathbb{P}, \mathbb{Q}, \ldots$ as objects and join-preserving maps $\widehat{\mathbb{P}} \rightarrow \widehat{\mathbb{Q}}$ as arrows. It turns out Lin has enough structure to be understood as a categorical model of Girard's linear logic [5, 23]. Accordingly, we'll call arrows of Lin linear maps.

Linear maps are represented by elements of $\widehat{\mathbb{P o p} \times \mathbb{Q}}$ and so by downwardsclosed subsets of the order $\mathbb{P}^{o p} \times \mathbb{Q}$. This relational presentation exposes an 
involution central in understanding $\mathbf{L i n}$ as a categorical model of classical linear logic. The involution of linear logic, yielding $\mathbb{P}^{\perp}$ on an object $\mathbb{P}$, is given by $\mathbb{P}^{o p}$; clearly, downwards-closed subsets of $\mathbb{P}^{\mathrm{op}} \times \mathbb{Q}$ correspond to downwards-closed subsets of $\left(\mathbb{Q}^{\text {op }}\right)^{\text {op }} \times \mathbb{P}^{\text {op }}$, showing how maps $\mathbb{P} \rightarrow \mathbb{Q}$ correspond to maps $\mathbb{Q}^{\perp} \rightarrow \mathbb{P}^{\perp}$ in Lin. The tensor product of $\mathbb{P}$ and $\mathbb{Q}$ is given by the product of preorders $\mathbb{P} \times \mathbb{Q}$; the singleton order $\mathbb{1}$ is a unit for tensor. Linear function space $\mathbb{P} \multimap \mathbb{Q}$ is then obtained as $\mathbb{P}$ op $\times \mathbb{Q}$. Products $\mathbb{P} \& \mathbb{Q}$ are given by $\mathbb{P}+\mathbb{Q}$, the disjoint juxtaposition of preorders. An element of $\widehat{\mathbb{P} \& \mathbb{Q}}$ can be identified with a pair $(X, Y)$ with $X \in \widehat{\mathbb{P}}$ and $Y \in \widehat{\mathbb{Q}}$, which provides the projections $\pi_{1}: \mathbb{P} \& \mathbb{Q} \rightarrow \mathbb{P}$ and $\pi_{2}: \mathbb{P} \& \mathbb{Q} \rightarrow \mathbb{Q}$ in Lin. More general, not just binary, products $\&_{i \in I} \mathbb{P}_{i}$ with projections $\pi_{j}$, for $j \in I$, are defined similarly. From the universal property of products, a collection of maps $f_{i}: \mathbb{P} \rightarrow \mathbb{P}_{i}$, for $i \in I$, can be tupled together to form a unique map $\left\langle f_{i}\right\rangle_{i \in I}: \mathbb{P} \rightarrow \&_{i \in I} \mathbb{P}_{i}$ with the property that $\pi_{j} \circ\left\langle f_{i}\right\rangle_{i \in I}=f_{j}$ for all $j \in I$. The empty product is given by the empty order $\mathbb{O}$ and, as the terminal object, is associated with unique maps $\varnothing_{\mathbb{P}}: \mathbb{P} \rightarrow \mathbb{O}$, constantly $\varnothing$, for any path order $\mathbb{P}$. All told, Lin is a $*$-autonomous category, so a symmetric monoidal closed category with a dualising object, and has finite products as required by Seely's definition of a model of linear logic [23].

In fact, Lin also has all coproducts, also given on objects $\mathbb{P}$ and $\mathbb{Q}$ by the juxtaposition $\mathbb{P}+\mathbb{Q}$ and so coinciding with products. Injection maps $i n_{1}: \mathbb{P} \rightarrow \mathbb{P}+\mathbb{Q}$ and $i n_{2}: \mathbb{Q} \rightarrow \mathbb{P}+\mathbb{Q}$ in Lin derive from the obvious injections into the disjoint sum of preorders. The empty coproduct is the empty order $\mathbb{O}$ which is then a zero object. This collapse of products and coproducts highlights that Lin has arbitrary biproducts. Via the isomorphism $\operatorname{Lin}(\mathbb{P}, \mathbb{Q}) \cong \widehat{\mathbb{P o p} \times \mathbb{Q}}$, each homset of Lin can be seen as a commutative monoid with neutral element the always $\varnothing$ map, itself written $\varnothing: \mathbb{P} \rightarrow \mathbb{Q}$, and sum given by union, written + . Composition in Lin is bilinear in that, given $f, f^{\prime}: \mathbb{P} \rightarrow \mathbb{Q}$ and $g, g^{\prime}: \mathbb{Q} \rightarrow \mathbb{R}$, we have $\left(g+g^{\prime}\right) \circ\left(f+f^{\prime}\right)=$ $g \circ f+g \circ f^{\prime}+g^{\prime} \circ f+g^{\prime} \circ f^{\prime}$. Further, given a family of objects $\left(\mathbb{P}_{\alpha}\right)_{\alpha \in A}$, we have for each $\beta \in A$ a diagram

$$
\mathbb{P}_{\beta} \underset{i n_{\beta}}{\stackrel{\pi_{\beta}}{\leftrightarrows}} \Sigma_{\alpha \in A} \mathbb{P}_{\alpha} \quad \text { such that } \begin{aligned}
\pi_{\beta} \circ i n_{\beta} & =1_{\mathbb{P}_{\beta}}, \\
\pi_{\beta} \circ i n_{\alpha} & =\varnothing \text { if } \alpha \neq \beta, \text { and } \\
\Sigma_{\alpha \in A}\left(i n_{\alpha} \circ \pi_{\alpha}\right) & =1_{\Sigma_{\alpha \in A} \mathbb{P}_{\alpha}} .
\end{aligned}
$$

Processes of type $\Sigma_{\alpha \in A} \mathbb{P}_{\alpha}$ may intuitively perform computation paths in any of the component path orders $\mathbb{P}_{\alpha}$.

We see that Lin is rich in structure. But linear maps alone are too restrictive. Being join-preserving, they in particular preserve the empty join. So, unlike e.g. prefixing, linear maps always send the inactive process $\varnothing$ 
to itself. Looking for a broader notion of maps between nondeterministic domains we follow the discipline of linear logic and consider non-linear maps, i.e. maps whose domain is under an exponential, !. One choice of a suitable exponential for Lin is got by taking $! \mathbb{P}$ to be the preorder obtained as the free finite-join completion of $\mathbb{P}$. Concretely, ! $\mathbb{P}$ can be defined to have finite subsets of $\mathbb{P}$ as elements with ordering given by $\preceq_{\mathbb{P}}$, defined for arbitrary subsets $X, Y$ of $\mathbb{P}$ as follows:

$$
X \preceq_{\mathbb{P}} Y \Longleftrightarrow \Longleftrightarrow_{\text {def }} \forall p \in X . \exists q \in Y . p \leq_{\mathbb{P}} q
$$

When $! \mathbb{P}$ is quotiented by the equivalence induced by the preorder we obtain a poset which is the free finite-join completion of $\mathbb{P}$. By further using the obvious inclusion of this completion into $\widehat{\mathbb{P}}$, we get a map $i_{\mathbb{P}}: ! \mathbb{P} \rightarrow \widehat{\mathbb{P}}$ sending a finite set $\left\{p_{1}, \ldots, p_{n}\right\}$ to the join $\mathrm{y}_{\mathbb{P}} p_{1}+\cdots+\mathrm{y}_{\mathbb{P}} p_{n}$. Such finite sums of primes are the finite (isolated, compact) elements of $\widehat{\mathbb{P}}$. The map $i_{\mathbb{P}}$ assumes the role of $\mathrm{y}_{\mathbb{P}}$ above. For any $X \in \widehat{\mathbb{P}}$ and $P \in ! \mathbb{P}$, we have $i_{\mathbb{P}} P \subseteq X$ iff $P \preceq_{\mathbb{P}} X$, and $X$ is the directed join of the finite elements below it:

$$
X=\bigcup_{P \preceq \mathbb{P} X} i_{\mathbb{P}} P .
$$

Further, $\widehat{\mathbb{P}}$ is the free directed-join completion of $! \mathbb{P}$ (also known as the ideal completion of $! \mathbb{P})$. This means that given any monotone map $f: ! \mathbb{P} \rightarrow$ $C$ for some directed-join complete poset $C$, there is a unique directed-join preserving (i.e. Scott continuous) map $f^{\ddagger}: \widehat{\mathbb{P}} \rightarrow C$ such that the diagram below commutes.

$$
! \mathbb{P} \underset{f}{\stackrel{i_{\mathbb{P}}}{\longrightarrow} \underset{C}{\widehat{\mathbb{P}}} \quad f^{\ddagger}} \quad f^{\ddagger} X=\bigcup_{P \preceq \mathbb{P} X} f P .
$$

Uniqueness of $f^{\ddagger}$, called the extension of $f$ along $i_{\mathbb{P}}$, follows from (6). As before, we can replace $C$ by a nondeterministic domain $\widehat{\mathbb{Q}}$ and by the freeness properties (2) and (7), there is a bijective correspondence between linear maps $! \mathbb{P} \rightarrow \mathbb{Q}$ and continuous maps $\widehat{\mathbb{P}} \rightarrow \widehat{\mathbb{Q}}$.

We define the category $\mathbf{C t s}$ to have path orders $\mathbb{P}, \mathbb{Q}, \ldots$ as objects and continuous maps $\widehat{\mathbb{P}} \rightarrow \widehat{\mathbb{Q}}$ as arrows. These arrows allow more process operations, including prefixing, to be expressed. The structure of Cts is induced by that of Lin via an adjunction between the two categories. 


\subsection{An Adjunction}

As linear maps are continuous, Cts has Lin as a sub-category, one which shares the same objects. We saw above that there is a bijection

$$
\operatorname{Lin}(! \mathbb{P}, \mathbb{Q}) \cong \operatorname{Cts}(\mathbb{P}, \mathbb{Q})
$$

This is in fact natural in $\mathbb{P}$ and $\mathbb{Q}$ so an adjunction with the inclusion Lin $\hookrightarrow$ Cts as right adjoint. Via $(7)$ the map $y ! \mathbb{P}: ! \mathbb{P} \rightarrow \widehat{\mathbb{P}}$ extends to a map $\eta_{\mathbb{P}}=\mathrm{y}_{! \mathbb{P}}^{\ddagger}: \mathbb{P} \rightarrow ! \mathbb{P}$ in Cts. Conversely, $i_{\mathbb{P}}: ! \mathbb{P} \rightarrow \widehat{\mathbb{P}}$ extends to a map $\varepsilon_{\mathbb{P}}=i_{\mathbb{P}}^{\dagger}: ! \mathbb{P} \rightarrow \mathbb{P}$ in Lin using (2). These maps are the unit and counit, respectively, of the adjunction:

$$
\begin{aligned}
\eta_{\mathbb{P}} X & =\bigcup_{P \preceq \mathbb{P}} X \mathrm{y} ! \mathbb{P} P & \varepsilon_{\mathbb{P}} X & =\bigcup_{P \in X} i_{\mathbb{P}} P \\
& =\left\{P \in ! \mathbb{P}: P \preceq_{\mathbb{P}} X\right\} & & =\{p \in \mathbb{P}: \exists P \in X . p \in P\}
\end{aligned}
$$

The left adjoint is the functor ! : Cts $\rightarrow$ Lin given on arrows $f: \mathbb{P} \rightarrow \mathbb{Q}$ by $\left(\eta_{\mathbb{Q}} \circ f \circ i_{\mathbb{P}}\right)^{\dagger}: ! \mathbb{P} \rightarrow ! \mathbb{Q}$. The bijection $(8)$ then maps $g: ! \mathbb{P} \rightarrow \mathbb{Q}$ in Lin to $\bar{g}=g \circ \eta_{\mathbb{P}}: \mathbb{P} \rightarrow \mathbb{Q}$ in Cts while its inverse maps $f: \mathbb{P} \rightarrow \mathbb{Q}$ in Cts to $\bar{f}=\varepsilon_{\mathbb{Q}} \circ$ ! $f$ in Lin. We call $\bar{g}$ and $\bar{f}$ the transpose of $g$ and $f$, respectively; of course, transposing twice yields back the original map. As Lin is a subcategory of Cts, the counit is also a map in Cts. We have $\varepsilon_{\mathbb{P}} \circ \eta_{\mathbb{P}}=1_{\mathbb{P}}$ and $X \subseteq \eta_{\mathbb{P}}\left(\varepsilon_{\mathbb{P}} X\right)$ for all $X \in \widehat{!} \mathbb{P}$.

Right adjoints preserve products, and so Cts has products given as in Lin. Hence, Cts is a symmetric monoidal category like Lin, and in fact, our adjunction is symmetric monoidal. In detail, there are isomorphisms of path orders,

$$
k: \mathbb{1} \cong ! \mathbb{O} \quad \text { and } \quad m_{\mathbb{P}, \mathbb{Q}}: ! \mathbb{P} \times ! \mathbb{Q} \cong !(\mathbb{P} \& \mathbb{Q})
$$

with $m_{\mathbb{P}, \mathbb{Q}}$ mapping a pair $(P, Q) \in ! \mathbb{P} \times ! \mathbb{Q}$ to the union $i n_{1} P \cup i n_{2} Q$; any element of $!(\mathbb{P} \& \mathbb{Q})$ can be written on this form. These isomorphisms induce isomorphisms with the same names in Lin with $m$ natural. Moreover, $k$ and $m$ commute with the associativity, symmetry and unit maps of Lin and Cts, such as $s_{\mathbb{P}, \mathbb{Q}}^{\text {Lin }}: \mathbb{P} \times \mathbb{Q} \cong \mathbb{Q} \times \mathbb{P}$ and $r_{\mathbb{Q}}^{\text {Cts }}: \mathbb{Q} \& \mathbb{O} \cong \mathbb{Q}$, making ! symmetric monoidal. It then follows [14] that the inclusion $\mathbf{L i n} \hookrightarrow \mathbf{C t s}$ is symmetric monoidal as well, and that the unit and counit are monoidal transformations. Thus, there are maps

$$
l: \mathbb{O} \rightarrow \mathbb{1} \quad \text { and } \quad n_{\mathbb{P}, \mathbb{Q}}: \mathbb{P} \& \mathbb{Q} \rightarrow \mathbb{P} \times \mathbb{Q}
$$

in Cts, with $n$ natural, corresponding to $k$ and $m$ above; $l$ maps $\varnothing$ to $\{*\}$ while $n_{\mathbb{P}, \mathbb{Q}}$ is the extension $h^{\ddagger}$ of the map $h\left(i n_{1} P \cup i n_{2} Q\right)=i_{\mathbb{P}} P \times i_{\mathbb{Q}} Q$. 
In addition, the unit makes the diagrams below commute and the counit satisfies similar properties.
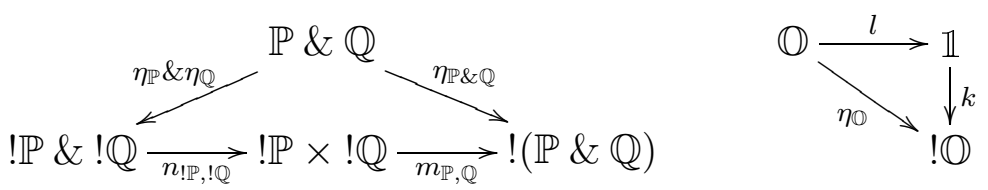

The diagram on the left can be written as $s \operatorname{st}_{\mathbb{P}, \mathbb{Q}} \circ\left(1_{\mathbb{P}} \& \eta_{\mathbb{Q}}\right)=\eta_{\mathbb{P} \& \mathbb{Q}}$ where $s t r$, the strength of ! viewed as a monad on Cts, is the natural transformation

$$
\mathbb{P} \& ! \mathbb{Q} \stackrel{\eta_{\mathbb{P}} \& 1_{\mathbb{Q}}}{\longrightarrow} ! \mathbb{P} \& ! \mathbb{Q} \stackrel{n_{! \mathbb{P}}, \mathbb{Q}}{\longrightarrow} ! \mathbb{P} \times ! \mathbb{Q} \stackrel{m_{\mathbb{P}, \mathbb{Q}}}{\longrightarrow} !(\mathbb{P} \& \mathbb{Q})
$$

Finally, recall that the category Lin is symmetric monoidal closed so that the functor $(\mathbb{Q} \multimap-)$ is right adjoint to $(-\times \mathbb{Q})$ for any object $\mathbb{Q}$. Together with the natural isomorphism $m$ this provides a right adjoint $(\mathbb{Q} \rightarrow-)$, defined by $(! \mathbb{Q} \multimap-)$, to the functor $(-\& \mathbb{Q})$ in Cts via the chain

$$
\begin{aligned}
\operatorname{Cts}(\mathbb{P} \& \mathbb{Q}, \mathbb{R}) & \cong \operatorname{Lin}(!(\mathbb{P} \& \mathbb{Q}), \mathbb{R}) \cong \operatorname{Lin}(! \mathbb{P} \times ! \mathbb{Q}, \mathbb{R}) \\
& \cong \operatorname{Lin}(! \mathbb{P}, ! \mathbb{Q} \multimap \mathbb{R}) \cong \operatorname{Cts}(\mathbb{P}, ! \mathbb{Q} \multimap \mathbb{R})=\operatorname{Cts}(\mathbb{P}, \mathbb{Q} \rightarrow \mathbb{R})
\end{aligned}
$$

-natural in $\mathbb{P}$ and $\mathbb{R}$. This demonstrates that $\mathbf{C t s}$ is cartesian closed, as is well known. The adjunction between Lin and Cts now satisfies the conditions put forward by Benton for a categorical model of intuitionistic linear logic, strengthening those of Seely [1, 23]; see also [14] for a recent survey of such models.

\section{Denotational Semantics}

HOPLA is directly suggested by the structure of Cts. The language is typed with types given by the grammar

$$
\mathbb{T}::=\mathbb{T}_{1} \rightarrow \mathbb{T}_{2}\left|\Sigma_{\alpha \in A} \mathbb{T}_{\alpha}\right| ! \mathbb{T}|T| \mu_{j} \vec{T} \cdot \overrightarrow{\mathbb{T}}
$$

The symbol $T$ is drawn from a set of type variables used in defining recursive types; closed type expressions are interpreted as path orders. Using vector notation, $\mu_{j} \vec{T} \cdot \overrightarrow{\mathbb{T}}$ abbreviates $\mu_{j} T_{1}, \ldots, T_{k} .\left(\mathbb{T}_{1}, \ldots, \mathbb{T}_{k}\right)$ and is interpreted as the $j$-component, for $1 \leq j \leq k$, of "the least" solution to the defining equations $T_{1}=\mathbb{T}_{1}, \ldots, T_{k}=\mathbb{T}_{k}$, in which the expressions $\mathbb{T}_{1}, \ldots, \mathbb{T}_{k}$ may contain the $T_{j}$ 's. We shall write $\mu \vec{T} . \overrightarrow{\mathbb{T}}$ as an abbreviation for the $k$-tuple with $j$-component $\mu_{j} \vec{T} . \overrightarrow{\mathbb{T}}$, and confuse a closed expression for a path order 
with the path order itself. Simultaneous recursive equations for path orders can be solved using information systems $[22,12]$. Here, it will be convenient to give a concrete, inductive characterisation based on a language of paths:

$$
p, q::=P \mapsto q|\beta p| P \mid \text { abs } p .
$$

Above, $P$ ranges over finite sets of paths. We use $P \mapsto q$ as notation for pairs in the function space $(! \mathbb{P})^{\mathrm{op}} \times \mathbb{Q}$. The language is complemented by formation rules using judgements $p: \mathbb{P}$, meaning that $p$ belongs to $\mathbb{P}$, displayed below on top of rules defining the ordering on $\mathbb{P}$ using judgements $p \leq \mathbb{P} p^{\prime}$. Recall that $P \preceq_{\mathbb{P}} P^{\prime}$ means $\forall p \in P . \exists p^{\prime} \in P^{\prime} . p \leq_{\mathbb{P}} p^{\prime}$.

$$
\begin{array}{cl}
\frac{P: ! \mathbb{P}}{P \mapsto \mathbb{Q}} & \frac{P^{\prime} \leq_{! \mathbb{P}} P \quad q \leq_{\mathbb{Q}} q^{\prime}}{P \mapsto q: \mathbb{P} \rightarrow \mathbb{Q}} \\
\frac{p: \mathbb{P}_{\beta} \quad \beta \in A}{\beta p: \Sigma_{\alpha \in A} \mathbb{P}_{\alpha}} & \frac{p \leq_{\mathbb{P}_{\beta}} p^{\prime}}{\beta p \leq_{\Sigma_{\alpha \in A} \mathbb{P}_{\alpha}} \beta p^{\prime}} \\
\frac{p_{1}: \mathbb{P} \cdots q_{n}: \mathbb{P}}{\left\{p_{1}, \ldots, p_{n}\right\}: ! \mathbb{P}} & \frac{P \preceq_{\mathbb{P}} P^{\prime}}{P \leq_{! \mathbb{P}} P^{\prime}} \\
\frac{p: \mathbb{T}_{j}[\mu \vec{T} \cdot \overrightarrow{\mathbb{T}} / \vec{T}]}{a b s p: \mu_{j} \vec{T} \cdot \overrightarrow{\mathbb{T}}} & \frac{p \leq_{\mathbb{T}_{j}[\mu \vec{T} \cdot \overrightarrow{\mathbb{T}} / \vec{T}]} p^{\prime}}{a b s p \leq_{\mu_{j} \vec{T} \cdot \overrightarrow{\mathbb{T}}} a b s p^{\prime}}
\end{array}
$$

Using information systems as in [12] yields the same representation, except for the tagging with abs in recursive types, done to help in the proof of adequacy in Sect. 4.1. So rather than the straight equality between a recursive type and its unfolding which we are used to from [12], we get an isomorphism abs : $\mathbb{T}_{j}[\mu \vec{T} \cdot \overrightarrow{\mathbb{T}} / \vec{T}] \cong \mu_{j} \vec{T} \cdot \overrightarrow{\mathbb{T}}$ whose inverse we call rep.

As an example, consider the type of CCS processes given in [19] as the path order $\mathbb{P}$ satisfying $\mathbb{P}=\Sigma_{\alpha \in A} ! \mathbb{P}$ where $A$ is a set of CCS actions. The elements of $\mathbb{P}$ then have the form abs $(\beta P)$ where $\beta \in A$ and $P$ is a finite set of paths from $\mathbb{P}$. Intuitively, a CCS process can perform such a path if it can perform the action $\beta$ and, following that, is able to perform each path in $P$.

The raw syntax of HOPLA terms is given by

$$
t, u::=x|\operatorname{rec} x . t| \Sigma_{i \in I} t_{i}|\lambda x . t| t u|\beta t| \pi_{\beta} t|! t|[u>! x \Rightarrow t] \mid \text { abs } t \mid \text { rep } t \text {. }
$$

The variables $x$ in the terms rec x.t, $\lambda$ x.t, and $[u>! x \Rightarrow t]$ are binding occurrences with scope $t$. We shall take for granted an understanding of free and bound variables, and substitution on raw terms.

Let $\mathbb{P}_{1}, \ldots, \mathbb{P}_{k}, \mathbb{Q}$ be closed type expressions and assume that the variables $x_{1}, \ldots, x_{k}$ are distinct. A syntactic judgement $x_{1}: \mathbb{P}_{1}, \ldots, x_{k}: \mathbb{P}_{k} \vdash t: \mathbb{Q}$ stands for a map $\llbracket x_{1}: \mathbb{P}_{1}, \ldots, x_{k}: \mathbb{P}_{k} \vdash t: \mathbb{Q} \rrbracket: \mathbb{P}_{1} \& \cdots \& \mathbb{P}_{k} \rightarrow \mathbb{Q}$ in Cts. 
We'll write $\Gamma$, or $\Lambda$, for an environment list $x_{1}: \mathbb{P}_{1}, \ldots, x_{k}: \mathbb{P}_{k}$ and most often abbreviate the denotation to $\mathbb{P}_{1} \& \cdots \& \mathbb{P}_{k} \stackrel{t}{\rightarrow} \mathbb{Q}$, or $\Gamma \stackrel{t}{\rightarrow} \mathbb{Q}$, or even $\llbracket t \rrbracket$, suppressing the typing information. When the environment list is empty, the corresponding product is the empty path order $\mathbb{O}$.

The term-formation rules are displayed below alongside their interpretations as constructors on maps of Cts, taking the maps denoted by the premises to that denoted by the conclusion (cf. [2]). We assume that the variables in any environment list which appears are distinct.

Structural rules. The rules handling environment lists are given as follows:

$$
\begin{aligned}
\overline{x: \mathbb{P} \vdash x: \mathbb{P}} & \stackrel{\mathbb{1}_{\mathbb{P}}}{\mathbb{P}} \\
\frac{\Gamma \vdash t: \mathbb{Q}}{\Gamma, x: \mathbb{P} \vdash t: \mathbb{Q}} & \frac{\Gamma \stackrel{t}{\rightarrow} \mathbb{Q}}{\Gamma \& \mathbb{P} \stackrel{t \& \varnothing_{\mathbb{P}}}{\longrightarrow} \mathbb{Q} \& \mathbb{O} \stackrel{r_{\mathbb{Q}}^{\text {Cts }}}{\longrightarrow} \mathbb{Q}} \\
\frac{\Gamma, y: \mathbb{Q}, x: \mathbb{P}, \Lambda \vdash t: \mathbb{R}}{\Gamma, x: \mathbb{P}, y: \mathbb{Q}, \Lambda \vdash t: \mathbb{R}} & \frac{\Gamma \& \mathbb{Q} \& \mathbb{P} \& \Lambda \stackrel{t}{\rightarrow} \mathbb{R}}{\Gamma \& \mathbb{P} \& \mathbb{Q} \& \Lambda \stackrel{t o\left(1_{\Gamma} \& s_{\mathbb{P}, \mathbb{Q}}^{\text {cts }} 1_{\Lambda}\right)}{\longrightarrow} \mathbb{R}} \\
\frac{\Gamma, x: \mathbb{P}, y: \mathbb{P} \vdash t: \mathbb{Q}}{\Gamma, z: \mathbb{P} \vdash t[z / x, z / y]: \mathbb{Q}} & \frac{\Gamma \& \mathbb{P} \& \mathbb{P} \stackrel{t}{\rightarrow} \mathbb{Q}}{\Gamma \& \mathbb{P} \stackrel{1_{\Gamma} \& \Delta_{\mathbb{P}}}{\longrightarrow} \& \mathbb{P} \& \mathbb{P} \stackrel{t}{\rightarrow} \mathbb{Q}}
\end{aligned}
$$

In the formation rule for contraction (22), the variable $z$ must be fresh; the map $\Delta_{\mathbb{P}}$ is the usual diagonal, given as $\left\langle 1_{\mathbb{P}}, 1_{\mathbb{P}}\right\rangle$.

Recursive definition. Since each $\widehat{\mathbb{P}}$ is a complete lattice, it admits least fixedpoints of continuous maps. If $f: \widehat{\mathbb{P}} \rightarrow \widehat{\mathbb{P}}$ is continuous, it has a least fixedpoint, fix $f \in \widehat{\mathbb{P}}$ obtained as $\bigcup_{n \in \omega} f^{n}(\varnothing)$. Below, fix $f$ is the fixpoint in $\operatorname{Cts}(\Gamma, \mathbb{P}) \cong \widehat{\Gamma \rightarrow \mathbb{P}}$ of the continuous operation $f$ mapping $g: \Gamma \rightarrow \mathbb{P}$ in Cts to the composition $\llbracket t \rrbracket \circ\left(1_{\Gamma} \& g\right) \circ \Delta_{\Gamma}$.

$$
\frac{\Gamma, x: \mathbb{P} \vdash t: \mathbb{P}}{\Gamma \vdash \text { rec } x . t: \mathbb{P}} \quad \frac{\Gamma \& \mathbb{P} \stackrel{t}{\rightarrow} \mathbb{P}}{\Gamma \stackrel{f i x f}{\longrightarrow} \mathbb{P}}
$$

Nondeterministic sum. Each path order $\mathbb{P}$ is associated with a join operation, $\Sigma: \&_{i \in I} \mathbb{P} \rightarrow \mathbb{P}$ in Cts taking a tuple $\left\langle t_{i}\right\rangle_{i \in I}$ to the join $\Sigma_{i \in I} t_{i}$ in $\widehat{\mathbb{P}}$. We'll write $\varnothing$ and $t_{1}+\cdots+t_{k}$ for finite sums.

$$
\frac{\Gamma \vdash t_{j}: \mathbb{P} \quad \text { all } j \in I}{\Gamma \vdash \Sigma_{i \in I} t_{i}: \mathbb{P}} \quad \frac{\Gamma \stackrel{t_{j}}{\longrightarrow} \mathbb{P} \text { all } j \in I}{\Gamma \stackrel{\left\langle t_{i}\right\rangle_{i \in I}}{\longrightarrow} \&_{i \in I} \mathbb{P} \stackrel{\Sigma}{\longrightarrow} \mathbb{P}}
$$

Function space. As noted at the end of Sect. 2.2, the category Cts is cartesian closed with function space $\mathbb{P} \rightarrow \mathbb{Q}$. Thus, there is a 1-1 correspondence curry 
from maps $\mathbb{P} \& \mathbb{Q} \rightarrow \mathbb{R}$ to maps $\mathbb{P} \rightarrow(\mathbb{Q} \rightarrow \mathbb{R})$ in Cts; its inverse is called uncurry. We obtain application, app $:(\mathbb{P} \rightarrow \mathbb{Q}) \& \mathbb{P} \rightarrow \mathbb{Q}$ as uncurry $\left(1_{\mathbb{P} \rightarrow \mathbb{Q}}\right)$.

$$
\begin{aligned}
\frac{\Gamma, x: \mathbb{P} \vdash t: \mathbb{Q}}{\Gamma \vdash \lambda x \cdot t: \mathbb{P} \rightarrow \mathbb{Q}} & \frac{\Gamma \& \mathbb{P} \stackrel{t}{\rightarrow} \mathbb{Q}}{\Gamma \stackrel{\text { curryt }}{\longrightarrow} \mathbb{P} \rightarrow \mathbb{Q}} \\
\frac{\Gamma \vdash t: \mathbb{P} \rightarrow \mathbb{Q} \Lambda \vdash u: \mathbb{P}}{\Gamma, \Lambda \vdash t u: \mathbb{Q}} & \frac{\Gamma \stackrel{t}{\rightarrow} \mathbb{P} \rightarrow \mathbb{Q} \Lambda \stackrel{u}{\rightarrow} \mathbb{P}}{\Gamma \& \Lambda \stackrel{t \& u}{\longrightarrow}(\mathbb{P} \rightarrow \mathbb{Q}) \& \mathbb{P} \stackrel{a p p}{\longrightarrow} \mathbb{Q}}
\end{aligned}
$$

Sum type. The category Cts does not have coproducts, but we can build a useful sum type out of the biproduct of Lin. The properties of (4) are obviously also satisfied in Cts, even though the construction is universal only in the subcategory of linear maps because composition is generally not bilinear in Cts. We'll write $\mathbb{O}$ and $\mathbb{P}_{1}+\cdots+\mathbb{P}_{k}$ for the empty and finite sum types. The product $\mathbb{P}_{1} \& \mathbb{P}_{2}$ of [19] with pairing $(t, u)$ and projection terms fst $t$, snd $t$ can be encoded, respectively, as the type $\mathbb{P}_{1}+\mathbb{P}_{2}$, and the terms $1 t+2 u$ and $\pi_{1} t, \pi_{2} t$.

$$
\begin{aligned}
\frac{\Gamma \vdash t: \mathbb{P}_{\beta} \quad \beta \in A}{\Gamma \vdash \beta t: \Sigma_{\alpha \in A} \mathbb{P}_{\alpha}} & \frac{\Gamma \stackrel{t}{\rightarrow} \mathbb{P}_{\beta} \beta \in A}{\Gamma \stackrel{t}{\rightarrow} \mathbb{P}_{\beta} \stackrel{i n_{\beta}}{\rightarrow} \Sigma_{\alpha \in A} \mathbb{P}_{\alpha}} \\
\frac{\Gamma \vdash t: \Sigma_{\alpha \in A} \mathbb{P}_{\alpha} \quad \beta \in A}{\Gamma \vdash \pi_{\beta} t: \mathbb{P}_{\beta}} & \frac{\Gamma \stackrel{t}{\rightarrow} \Sigma_{\alpha \in A} \mathbb{P}_{\alpha} \quad \beta \in A}{\Gamma \stackrel{t}{\rightarrow} \Sigma_{\alpha \in A} \mathbb{P}_{\alpha} \stackrel{\pi_{\beta}}{\longrightarrow} \mathbb{P}_{\beta}}
\end{aligned}
$$

Prefixing. The adjunction between Lin and $\mathbf{C t s}$ provides a type constructor, $!(-)$, for which the unit $\eta_{\mathbb{P}}: \mathbb{P} \rightarrow ! \mathbb{P}$ and counit $\varepsilon_{\mathbb{P}}: ! \mathbb{P} \rightarrow \mathbb{P}$ may interpret term constructors and deconstructors, respectively. The behaviour of $\eta_{\mathbb{P}}$ with respect to maps of $\mathbf{C t s}$ fits that of an anonymous prefix operation. We'll say that $\eta_{\mathbb{P}}$ maps $u$ of type $\mathbb{P}$ to a "prefixed" process $! u$ of type ! $\mathbb{P}$; intuitively, the process ! $u$ will be able to perform an action, which we call !, before continuing as $u$.

$$
\frac{\Gamma \vdash u: \mathbb{P}}{\Gamma \vdash ! u: ! \mathbb{P}} \quad \frac{\Gamma \stackrel{u}{\rightarrow} \mathbb{P}}{\Gamma \stackrel{u}{\rightarrow} \mathbb{P} \stackrel{\eta_{\mathbb{P}}}{\longrightarrow} ! \mathbb{P}}
$$

By the universal property of $\eta_{\mathbb{P}}$, if $t$ of type $\mathbb{Q}$ has a free variable of type $\mathbb{P}$, and so is interpreted as a map $t: \mathbb{P} \rightarrow \mathbb{Q}$ in Cts, then the transpose $\bar{t}=\varepsilon_{\mathbb{Q}} \circ ! t$ is the unique map $! \mathbb{P} \rightarrow \mathbb{Q}$ in Lin such that $t=\bar{t} \circ \eta_{\mathbb{P}}$. With $u$ of type $! \mathbb{P}$, we'll write $[u>! x \Rightarrow t]$ for $\bar{t} u$. Intuitively, this construction "tests" or matches $u$ against the pattern $! x$ and passes the results of successful matches for $x$ on to $t$. Indeed, first prefixing a term $u$ of type $\mathbb{P}$ and then matching yields a successful match $u$ for $x$ as $\bar{t}\left(\eta_{\mathbb{P}} u\right)=t u$. By linearity of $\bar{t}$, the possibly multiple results of successful matches are nondeterministically 
summed together; the denotations of $\left[\Sigma_{i \in I} u_{i}>! x \Rightarrow t\right]$ and $\Sigma_{i \in I}\left[u_{i}>! x \Rightarrow t\right]$ are identical.

The above clearly generalises to the case where $u$ is an open term, but if $t$ has free variables other than $x$, we need to make use of the strength map (13):

$$
\frac{\Gamma, x: \mathbb{P} \vdash t: \mathbb{Q} \Lambda \vdash u: ! \mathbb{P}}{\Gamma, \Lambda \vdash[u>! x \Rightarrow t]: \mathbb{Q}} \quad \frac{\Gamma \& \mathbb{P} \stackrel{t}{\rightarrow} \mathbb{Q} \Lambda \stackrel{u}{\rightarrow} ! \mathbb{P}}{\Gamma \& \Lambda \stackrel{1_{\Gamma} \& u}{\longrightarrow} \Gamma \& ! \mathbb{P} \stackrel{\operatorname{str}_{\Gamma, \mathbb{P}}}{\longrightarrow} !(\Gamma \& \mathbb{P}) \stackrel{\bar{t}}{\rightarrow} \mathbb{Q}}
$$

Recursive types. Folding and unfolding recursive types is accompanied by term constructors abs and rep:

$$
\begin{array}{cc}
\frac{\Gamma \vdash t: \mathbb{T}_{j}[\mu \vec{T} \cdot \overrightarrow{\mathbb{T}} / \vec{T}]}{\Gamma \vdash a b s t: \mu_{j} \vec{T} \cdot \overrightarrow{\mathbb{T}}} & \frac{\Gamma \stackrel{t}{\rightarrow} \mathbb{T}_{j}[\mu \vec{T} \cdot \overrightarrow{\mathbb{T}} / \vec{T}]}{\Gamma \stackrel{t}{\rightarrow} \mathbb{T}_{j}[\mu \vec{T} \cdot \overrightarrow{\mathbb{T}} / \vec{T}] \stackrel{a b s}{\longrightarrow} \mu_{j} \vec{T} \cdot \overrightarrow{\mathbb{T}}} \\
\frac{\Gamma \vdash t: \mu_{j} \vec{T} \cdot \overrightarrow{\mathbb{T}}}{\Gamma \vdash \operatorname{rept}: \mathbb{T}_{j}[\mu \vec{T} \cdot \overrightarrow{\mathbb{T}} / \vec{T}]} & \frac{\Gamma \stackrel{t}{\rightarrow} \mu_{j} \vec{T} \cdot \overrightarrow{\mathbb{T}}}{\Gamma \stackrel{t}{\rightarrow} \mu_{j} \vec{T} \cdot \overrightarrow{\mathbb{T}} \stackrel{\text { rep }}{\longrightarrow} \mathbb{T}_{j}[\mu \vec{T} \cdot \overrightarrow{\mathbb{T}} / \vec{T}]}
\end{array}
$$

\subsection{Useful Equivalences}

We provide some technical results about the path semantics which are used in the proof of soundness, Proposition 4.3. Proofs can be found in [20].

Lemma 3.1 (Substitution) Suppose $\Gamma, x: \mathbb{P} \vdash t: \mathbb{Q}$ and $\Lambda \vdash u: \mathbb{P}$ with $\Gamma$ and $\Lambda$ disjoint. Then $\Gamma, \Lambda \vdash t[u / x]: \mathbb{Q}$ with denotation given by the composition $\llbracket t \rrbracket \circ\left(1_{\Gamma} \& \llbracket u \rrbracket\right)$.

Corollary 3.2 If $\Gamma, x: \mathbb{P} \vdash t: \mathbb{P}$, then $\Gamma \vdash t[$ rec x.t $/ x]: \mathbb{P}$ and $\llbracket$ rec x.t $\rrbracket=$ $\llbracket t[$ rec $x . t / x] \rrbracket$ so recursion amounts to unfolding.

Corollary 3.3 Application amounts to substitution. In the situation of the substitution lemma, we have $\llbracket(\lambda x . t) u \rrbracket=\llbracket t[u / x] \rrbracket$.

Proposition 3.4 From the properties of the biproduct we get:

$$
\begin{aligned}
\llbracket \pi_{\beta}(\beta t) \rrbracket & =\llbracket t \rrbracket \\
\llbracket \pi_{\alpha}(\beta t) \rrbracket & =\varnothing \quad \text { if } \alpha \neq \beta \\
\llbracket \Sigma_{\alpha \in A} \alpha\left(\pi_{\alpha}(t)\right) \rrbracket & =\llbracket t \rrbracket \quad \text { where } \Gamma \vdash t: \Sigma_{\alpha \in A} \mathbb{P}_{\alpha}
\end{aligned}
$$

In addition, $\llbracket \beta\left(\sum_{i \in I} t_{i}\right) \rrbracket=\llbracket \Sigma_{i \in I}\left(\beta t_{i}\right) \rrbracket$ and $\llbracket \pi_{\beta}\left(\sum_{i \in I} t_{i}\right) \rrbracket=\llbracket \Sigma_{i \in I}\left(\pi_{\beta} t_{i}\right) \rrbracket$ by linearity of injection and projection. 
Proposition 3.5 The prefix match satisfies the properties

$$
\begin{aligned}
\llbracket[! u>! x \Rightarrow t] \rrbracket & =\llbracket t[u / x] \rrbracket \\
\llbracket\left[\Sigma_{i \in I} u_{i}>! x \Rightarrow t\right] \rrbracket & =\llbracket \Sigma_{i \in I}\left[u_{i}>! x \Rightarrow t\right] \rrbracket
\end{aligned}
$$

Proposition 3.6 As abs and rep are inverses and linear, we get

$$
\begin{aligned}
& \llbracket r e p(a b s t) \rrbracket=\llbracket t \rrbracket \quad \llbracket a b s\left(\sum_{i \in I} t_{i}\right) \rrbracket=\llbracket \Sigma_{i \in I}\left(a b s t_{i}\right) \rrbracket \\
& \llbracket a b s(r e p t) \rrbracket=\llbracket t \rrbracket \quad \llbracket r e p\left(\Sigma_{i \in I} t_{i}\right) \rrbracket=\llbracket \Sigma_{i \in I}\left(r e p t_{i}\right) \rrbracket
\end{aligned}
$$

\subsection{Full Abstraction}

We define a program to be a closed term $t$ of type !OO. A $(\Gamma, \mathbb{P})$-program context $C$ is a term with holes into which a term $t$ with $\Gamma \vdash t: \mathbb{P}$ may be put to form a program $\vdash C(t):$ ! $\mathbb{O}$. The denotational semantics gives rise to a type-respecting contextual preorder [16]:

Definition 3.7 Suppose $\Gamma \vdash t_{1}: \mathbb{P}$ and $\Gamma \vdash t_{2}: \mathbb{P}$. We say that $t_{1}$ and $t_{2}$ are related by contextual preorder, written $t_{1} \sqsubset t_{2}$, iff for all $(\Gamma, \mathbb{P})$-program contexts $C$, we have $\llbracket C\left(t_{1}\right) \rrbracket \neq \varnothing \Longrightarrow \llbracket C\left(t_{2}\right) \rrbracket \neq \varnothing$. If both $t_{1} \sqsubseteq t_{2}$ and $t_{2} \sqsubseteq t_{1}$, we say that $t_{1}$ and $t_{2}$ are contextually equivalent.

Contextual equivalence coincides with path equivalence as do the associated preorders:

Theorem 3.8 (Full Abstraction) Suppose $\Gamma \vdash t_{1}: \mathbb{P}$ and $\Gamma \vdash t_{2}: \mathbb{P}$. Then

$$
\llbracket t_{1} \rrbracket \subseteq \llbracket t_{2} \rrbracket \Longleftrightarrow t_{1} \sqsubseteq t_{2}
$$

Proof. Suppose $\llbracket t_{1} \rrbracket \subseteq \llbracket t_{2} \rrbracket$ and let $C$ be a $(\Gamma, \mathbb{P})$-program context with $\llbracket C\left(t_{1}\right) \rrbracket \neq \varnothing$. As $\llbracket t_{1} \rrbracket \subseteq \llbracket t_{2} \rrbracket$ we have $\llbracket C\left(t_{2}\right) \rrbracket \neq \varnothing$ by compositionality and monotonicity, and so $t_{1} \sqsubseteq t_{2}$ as wanted.

To prove the converse we define for each path $p: \mathbb{P}$ a closed term $t_{p}$ of type $\mathbb{P}$ and a $(\mathbb{O}, \mathbb{P})$-program context $C_{p}$ that respectively "realise" and "consume" the path $p$, by induction on the structure of $p .{ }^{1}$

$$
\begin{array}{rlrl}
t_{P \mapsto q} & \equiv_{\text {def }} \lambda x \cdot\left[C_{P}^{\prime}(x)>! x^{\prime} \Rightarrow t_{q}\right] & C_{P \mapsto q} & \equiv_{\text {def }} C_{q}\left(-t_{P}^{\prime}\right) \\
t_{\beta p} & \equiv_{\text {def }} \beta t_{p} & C_{\beta p} & \equiv_{\text {def }} C_{p}\left(\pi_{\beta}-\right) \\
t_{P} & \equiv_{\text {def }} ! t_{P}^{\prime} & C_{P} \equiv_{\operatorname{def}}\left[->! x \Rightarrow C_{P}^{\prime}(x)\right] \\
t_{a b s p} & \equiv_{\text {def }} \text { abs } t_{p} & C_{a b s p} & \equiv_{\operatorname{def}} C_{p}(\text { rep }-)
\end{array}
$$

\footnotetext{
${ }^{1}$ We have recently become aware that this technique has been applied by Guy McCusker to prove full abstraction for a version of Idealized Algol [13].
} 
Here, $t_{P}^{\prime}$ and $C_{P}^{\prime}$ realise and consume finite sets of paths:

$$
\begin{aligned}
t_{\left\{p_{1}, \ldots, p_{n}\right\}}^{\prime} & \equiv_{\text {def }} t_{p_{1}}+\cdots+t_{p_{n}} \\
C_{\left\{p_{1}, \ldots, p_{n}\right\}}^{\prime} & \equiv_{\text {def }}\left[C_{p_{1}}>! x_{1} \Rightarrow \cdots \Rightarrow\left[C_{p_{n}}>! x_{n} \Rightarrow ! \varnothing\right] \cdots\right]
\end{aligned}
$$

Note that $t_{\varnothing}^{\prime} \equiv \varnothing$ and $C_{\varnothing}^{\prime} \equiv ! \varnothing$. Although the syntax of $t_{P}^{\prime}$ and $C_{P}^{\prime}$ depends on a choice of permutation of the elements of $P$, the semantics obtained for different permutations is the same. Indeed, we have ( $z$ being a fresh variable):

$$
\begin{aligned}
& \llbracket t_{p} \rrbracket=\mathrm{y}_{\mathbb{P}} p \quad \llbracket \lambda z \cdot C_{p}(z) \rrbracket=\mathrm{y}_{\mathbb{P} \rightarrow ! \mathbb{O}}(\{p\} \mapsto \varnothing) \\
& \llbracket t_{P}^{\prime} \rrbracket=i_{\mathbb{P}} P \quad \llbracket \lambda z \cdot C_{P}^{\prime}(z) \rrbracket=\mathrm{y}_{\mathbb{P} \rightarrow ! \mathbb{O}}(P \mapsto \varnothing)
\end{aligned}
$$

It then follows from the substitution lemma that for any $p: \mathbb{P}$ and $\vdash t: \mathbb{P}$,

$$
p \in \llbracket t \rrbracket \Longleftrightarrow \llbracket C_{p}(t) \rrbracket \neq \varnothing .
$$

Suppose $t_{1} \sqsubseteq t_{2}$ with $t_{1}$ and $t_{2}$ closed. Given any $p \in \llbracket t_{1} \rrbracket$ we have $\llbracket C_{p}\left(t_{1}\right) \rrbracket \neq$ $\varnothing$ and so using $t_{1} \sqsubset t_{2}$, we get $\llbracket C_{p}\left(t_{2}\right) \rrbracket \neq \varnothing$, so that $p \in \llbracket t_{2} \rrbracket$. It follows that $\llbracket t_{1} \rrbracket \subseteq \llbracket t_{2} \rrbracket$.

As for open terms, suppose $\Gamma \equiv x_{1}: \mathbb{P}_{1}, \ldots, x_{k}: \mathbb{P}_{k}$. Writing $\lambda \vec{x} . t_{1}$ for the closed term $\lambda x_{1} \cdots \lambda x_{k} . t_{1}$ and likewise for $t_{2}$, we get

$$
\begin{aligned}
t_{1} \sqsubseteq t_{2} & \Longrightarrow \lambda \vec{x} \cdot t_{1} \sqsubseteq \lambda \vec{x} \cdot t_{2} \\
& \Longrightarrow \llbracket \lambda \vec{x} \cdot t_{1} \rrbracket \subseteq \llbracket \lambda \vec{x} \cdot t_{2} \rrbracket \\
& \Longrightarrow \llbracket t_{1} \rrbracket \subseteq \llbracket t_{2} \rrbracket
\end{aligned}
$$

The proof is complete.

\section{Operational Semantics}

HOPLA can be given an operational semantics using actions defined by

$$
a::=u \mapsto a|\beta a| ! \mid a b s a .
$$

We assign types to actions a using a judgement of the form $\mathbb{P}: a: \mathbb{P}^{\prime}$. Intuitively, performing the action $a$ turns a process of type $\mathbb{P}$ into a process of type $\mathbb{P}^{\prime}$.

$$
\begin{gathered}
\frac{\vdash u: \mathbb{P} \quad \mathbb{Q}: a: \mathbb{P}^{\prime}}{\mathbb{P} \rightarrow \mathbb{Q}: u \mapsto a: \mathbb{P}^{\prime}} \quad \frac{\mathbb{P}_{\beta}: a: \mathbb{P}^{\prime} \quad \beta \in A}{\Sigma_{\alpha \in A} \mathbb{P}_{\alpha}: \beta a: \mathbb{P}^{\prime}} \\
\frac{\mathbb{T}_{j}[\mu \vec{T} \cdot \overrightarrow{\mathbb{T}} / \vec{T}]: a: \mathbb{P}^{\prime}}{! \mathbb{P}: !: \mathbb{P}} \quad \frac{\mu_{j} \vec{T} \cdot \overrightarrow{\mathbb{T}}: a b s a: \mathbb{P}^{\prime}}{\mu}
\end{gathered}
$$




$$
\begin{array}{cc}
\frac{\mathbb{P}: t[\text { rec } x . t / x] \stackrel{a}{\rightarrow} t^{\prime}}{\mathbb{P}: \text { rec } x . t \stackrel{a}{\rightarrow} t^{\prime}} & \frac{\mathbb{P}: t_{j} \stackrel{a}{\rightarrow} t^{\prime}}{\mathbb{P}: \Sigma_{i \in I} t_{i} \stackrel{a}{\rightarrow} t^{\prime}} j \in I \\
\frac{\mathbb{Q}: t[u / x] \stackrel{a}{\rightarrow} t^{\prime}}{\mathbb{P} \rightarrow \mathbb{Q}: \lambda x . t \stackrel{u \mapsto a}{\rightarrow} t^{\prime}} & \frac{\mathbb{P} \rightarrow \mathbb{Q}: t \stackrel{u \mapsto a}{\rightarrow} t^{\prime}}{\mathbb{Q}: t u \stackrel{a}{\rightarrow} t^{\prime}} \\
\frac{\mathbb{P}_{\beta}: t \stackrel{a}{\rightarrow} t^{\prime}}{\Sigma_{\alpha \in A} \mathbb{P}_{\alpha}: \beta t \stackrel{\beta a}{\rightarrow} t^{\prime}} & \frac{\Sigma_{\alpha \in A} \mathbb{P}_{\alpha}: t \stackrel{\beta a}{\rightarrow} t^{\prime}}{\mathbb{P}_{\beta}: \pi_{\beta} t \stackrel{a}{\rightarrow} t^{\prime}} \\
\frac{! \mathbb{P}: u \stackrel{!}{\rightarrow} u^{\prime} \quad \mathbb{Q}: t\left[u^{\prime} / x\right] \stackrel{a}{\rightarrow} t^{\prime}}{\mathbb{Q}:[u>! x \Rightarrow t] \stackrel{a}{\rightarrow} t^{\prime}} \\
\frac{\mathbb{T}_{j}[\mu \vec{T} \cdot \overrightarrow{\mathbb{T}} / \vec{T}]: t \stackrel{a}{\rightarrow} t^{\prime}}{\mu_{j} \vec{T} \cdot \overrightarrow{\mathbb{T}}: a b s t \stackrel{\text { abs a }}{\rightarrow} t^{\prime}} \quad \frac{\mu_{j} \vec{T} \cdot \overrightarrow{\mathbb{T}}: t \stackrel{a b s a}{\rightarrow} t^{\prime}}{\mathbb{T}_{j}[\mu \vec{T} \cdot \overrightarrow{\mathbb{T}} / \vec{T}]: \operatorname{rep} t \stackrel{a}{\rightarrow} t^{\prime}}
\end{array}
$$

Figure 1: Operational rules

Notice that in $\mathbb{P}: a: \mathbb{P}^{\prime}$, the type $\mathbb{P}^{\prime}$ is unique given $\mathbb{P}$ and $a$. The operational rules of Fig. 1 define a relation $\mathbb{P}: t \stackrel{a}{\rightarrow} t^{\prime}$ where $\vdash t: \mathbb{P}$ and $\mathbb{P}: a: \mathbb{P}^{\prime} .^{2}$ The operational rules are type-correct:

Proposition 4.1 If $\mathbb{P}: t \stackrel{a}{\rightarrow} t^{\prime}$ with $\mathbb{P}: a: \mathbb{P}^{\prime}$, then $\vdash t^{\prime}: \mathbb{P}^{\prime}$.

Proof. By rule-induction on the operational rules.

Abstraction. Suppose $\mathbb{P} \rightarrow \mathbb{Q}: \lambda x . t \stackrel{u \mapsto a}{\longrightarrow} t^{\prime}$ is derived from $\mathbb{Q}: t[u / x] \stackrel{a}{\rightarrow} t^{\prime}$ with $\mathbb{P} \mapsto \mathbb{Q}: u \mapsto a: \mathbb{P}^{\prime}$. By typing of actions, we have $\vdash u: \mathbb{P}$ and $\mathbb{Q}: a: \mathbb{P}^{\prime}$. The induction hypothesis then yields $\vdash t^{\prime}: \mathbb{P}^{\prime}$ as wanted. Note also that the substitution $t[u / x]$ is well-formed as $x: \mathbb{P} \vdash t: \mathbb{Q}$ follows from $\vdash \lambda x . t: \mathbb{P} \rightarrow \mathbb{Q}$ by the typing rules.

Application. Suppose $\mathbb{Q}: t u \stackrel{a}{\rightarrow} t^{\prime}$ is derived from $\mathbb{P} \rightarrow \mathbb{Q}: t \stackrel{u \mapsto a}{\rightarrow} t^{\prime}$ with $\mathbb{Q}: a: \mathbb{P}^{\prime}$. By the premise and typing rules, we have $\vdash t: \mathbb{P} \rightarrow \mathbb{Q}$ and $\vdash u: \mathbb{P}$, such that $\mathbb{P} \rightarrow \mathbb{Q}: u \mapsto a: \mathbb{P}^{\prime}$. The induction hypothesis then yields $\vdash t^{\prime}: \mathbb{P}^{\prime}$ as wanted.

Prefixing. Suppose $! \mathbb{P}: ! t \stackrel{!}{\rightarrow} t$ with $! \mathbb{P}: !: \mathbb{P}$. Then $\vdash ! t: ! \mathbb{P}$ and so by the typing rules, $\vdash t: \mathbb{P}$ as wanted.

Prefix match. Suppose $\mathbb{Q}:[u>! x \Rightarrow t] \stackrel{a}{\rightarrow} t^{\prime}$ is derived from $! \mathbb{P}: u \stackrel{!}{\rightarrow} u^{\prime}$ and $\mathbb{Q}: t\left[u^{\prime} / x\right] \stackrel{a}{\rightarrow} t^{\prime}$ with $\mathbb{Q}: a: \mathbb{P}^{\prime}$. By the induction hypothesis applied to the right premise, we get $\vdash t^{\prime}: \mathbb{P}^{\prime}$ as wanted. Note also that we have $\vdash u: ! \mathbb{P}$ and

\footnotetext{
${ }^{2}$ The explicit types in the operational rules were missing in the rules given in [19]. They are needed to ensure that the types of $t$ and $a$ agree in transitions.
} 
therefore $\vdash u^{\prime}: \mathbb{P}$ by the induction hypothesis for the left premise. Thus, as $x: \mathbb{P} \vdash t: \mathbb{Q}$, the substitution $t\left[u^{\prime} / x\right]$ is well-formed.

The remaining cases are handled similarly.

Accordingly, we'll write $\mathbb{P}: t \stackrel{a}{\rightarrow} t^{\prime}: \mathbb{P}^{\prime}$ when $\mathbb{P}: t \stackrel{a}{\rightarrow} t^{\prime}$ and $\mathbb{P}: a: \mathbb{P}^{\prime}$.

\subsection{Soundness and Adequacy}

For each action $\mathbb{P}: a: \mathbb{P}^{\prime}$ we define a linear map $a^{*}: \mathbb{P} \rightarrow ! \mathbb{P}^{\prime}$ which intuitively maps a process $t$ of type $\mathbb{P}$ to a representation of its possible successors after performing the action $a$. In order to distinguish between, say, the successor $\varnothing$ and no successors, $a^{*}$ embeds into the type $! \mathbb{P}^{\prime}$ rather than using $\mathbb{P}^{\prime}$ itself. For instance, the successors after action! of the processes ! $\varnothing$ and $\varnothing$ are,

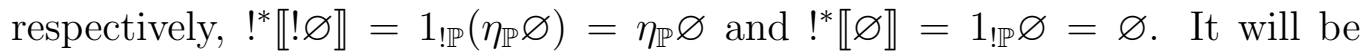
convenient to treat $a^{*}$ as a syntactic operation and so we define a term $a^{*} t$ such that $\llbracket a^{*} t \rrbracket=a^{*} \llbracket t \rrbracket$ :

$$
\begin{aligned}
(u \mapsto a)^{*} & =a^{*} \circ \text { app } \circ(-\& \llbracket u \rrbracket) & (u \mapsto a)^{*} t & \equiv a^{*}(t u) \\
(\beta a)^{*} & =a^{*} \circ \pi_{\beta} & (\beta a)^{*} t & \equiv a^{*}\left(\pi_{\beta} t\right) \\
!^{*} & =1_{! \mathbb{P}} & !^{*} t & \equiv t \\
(\text { abs } a)^{*} & =a^{*} \circ \text { rep } & (\text { abs a })^{*} t & \equiv a^{*}(\text { rep } t)
\end{aligned}
$$

The role of $a^{*}$ is to reduce the action $a$ to a prefix action:

Lemma $4.2 \mathbb{P}: t \stackrel{a}{\rightarrow} t^{\prime}: \mathbb{P}^{\prime} \Longleftrightarrow ! \mathbb{P}^{\prime}: a^{*} t \stackrel{!}{\rightarrow} t^{\prime}: \mathbb{P}^{\prime}$

Proof. By structural induction on $a$ exploiting the fact that there is only one operational rule deriving transitions from each of the constructs application $t u$, injection $\beta t$, and folding abst so that

$$
\begin{aligned}
& \mathbb{P} \rightarrow \mathbb{Q}: t \stackrel{u \mapsto a}{\longrightarrow} t^{\prime}: \mathbb{P}^{\prime} \Longleftrightarrow \mathbb{Q}: t u \stackrel{a}{\rightarrow} t^{\prime}: \mathbb{P}^{\prime} \\
& \Sigma_{\alpha \in A} \mathbb{P}_{\alpha}: t \stackrel{\beta a}{\longrightarrow} t^{\prime}: \mathbb{P}^{\prime} \Longleftrightarrow \mathbb{P}_{\beta}: \pi_{\beta} t \stackrel{a}{\rightarrow} t^{\prime}: \mathbb{P}^{\prime} \\
& \mu_{j} \vec{T} \cdot \overrightarrow{\mathbb{T}}: t \stackrel{\text { abs } a}{\longrightarrow} t^{\prime}: \mathbb{P}^{\prime} \Longleftrightarrow \mathbb{T}_{j}[\mu \vec{T} \cdot \overrightarrow{\mathbb{T}} / \vec{T}]: \operatorname{rep} t \stackrel{a}{\rightarrow} t^{\prime}: \mathbb{P}^{\prime}
\end{aligned}
$$

Function space. We argue as follows:

$$
\begin{array}{rlrl}
\mathbb{P} & \rightarrow \mathbb{Q}: t \stackrel{u \mapsto a}{\longrightarrow} t^{\prime}: \mathbb{P}^{\prime} & \\
& \Longleftrightarrow \mathbb{Q}: t u \stackrel{a}{\rightarrow} t^{\prime}: \mathbb{P}^{\prime} & & \text { (by }(45)) \\
& \Longleftrightarrow ! \mathbb{P}^{\prime}: a^{*}(t u) \stackrel{!}{\rightarrow} t^{\prime}: \mathbb{P}^{\prime} & & \text { (ind. hyp.) } \\
& \Longleftrightarrow ! \mathbb{P}^{\prime}:(u \mapsto a)^{*} t \stackrel{!}{\rightarrow} t^{\prime}: \mathbb{P}^{\prime} & & \text { (def. of } \left.(u \mapsto a)^{*} t\right)
\end{array}
$$


Sum. We argue as follows:

$$
\begin{array}{lll}
\Sigma_{\alpha \in A} \mathbb{P}_{\alpha}: t \stackrel{\beta a}{\longrightarrow} t^{\prime}: \mathbb{P}^{\prime} & \\
& \Longleftrightarrow \mathbb{P}_{\beta}: \pi_{\beta} t \stackrel{a}{\rightarrow} t^{\prime}: \mathbb{P}^{\prime} & \\
& \Longleftrightarrow ! \mathbb{P}^{\prime}: a^{*}\left(\pi_{\beta} t\right) \stackrel{!}{\rightarrow} t^{\prime}: \mathbb{P}^{\prime} & \text { (ind. hyp.) } \\
& \Longleftrightarrow ! \mathbb{P}^{\prime}:(\beta a)^{*} t \stackrel{!}{\rightarrow} t^{\prime}: \mathbb{P}^{\prime} & \text { (def. of } \left.(\beta a)^{*} t\right)
\end{array}
$$

Prefix. We argue as follows:

$$
\begin{aligned}
& ! \mathbb{P}: t \stackrel{!}{\rightarrow} t^{\prime}: \mathbb{P} \\
& \quad \Longleftrightarrow ! \mathbb{P}: !^{*} t \stackrel{!}{\rightarrow} t^{\prime}: \mathbb{P} \quad\left(\text { def. of } !^{*} t\right)
\end{aligned}
$$

Recursion. We argue as follows:

$$
\begin{array}{lll}
\mu_{j} \vec{T} \cdot \overrightarrow{\mathbb{T}}: t \stackrel{\text { abs a }}{\longrightarrow} t^{\prime}: \mathbb{P}^{\prime} & \\
& \Longleftrightarrow \mathbb{T}_{j}[\mu \vec{T} \cdot \overrightarrow{\mathbb{T}} / \vec{T}]: \operatorname{rep} t \stackrel{a}{\rightarrow} t^{\prime}: \mathbb{P}^{\prime} & (\text { by }(45)) \\
& \Longleftrightarrow ! \mathbb{P}^{\prime}: a^{*}(\text { rep } t) \stackrel{!}{\rightarrow} t^{\prime}: \mathbb{P}^{\prime} & \text { (ind. hyp.) } \\
\Longleftrightarrow ! \mathbb{P}^{\prime}:(\text { abs } a)^{*} t \stackrel{!}{\rightarrow} t^{\prime}: \mathbb{P}^{\prime} & \text { (def. of } \left.(\text { abs } a)^{*} t\right)
\end{array}
$$

The structural induction is complete.

Note that the reduction is done uniformly at all types using deconstructor contexts: application, projection, and unfolding. This explains the somewhat mysterious function space actions $u \mapsto a$. A similar use of labels to carry context information appears e.g. in [6].

Soundness says that the operational notion of "successor" is included in the semantic notion:

Proposition 4.3 (Soundness) If $\mathbb{P}: t \stackrel{a}{\rightarrow} t^{\prime}: \mathbb{P}^{\prime}$, then $\eta_{\mathbb{P}^{\prime}} \llbracket t^{\prime} \rrbracket \subseteq a^{*} \llbracket t \rrbracket$.

Proof. By rule-induction on the transition rules. We'll dispense with the typing information in transitions for clarity.

Recursive definition. Suppose rec x.t $\stackrel{a}{\rightarrow} t^{\prime}$ is derived from $t[$ rec x.t $/ x] \stackrel{a}{\rightarrow} t^{\prime}$. By the induction hypothesis and Corollary 3.2,

$$
\llbracket ! t^{\prime} \rrbracket \subseteq a^{*} \llbracket t[\operatorname{rec} x . t / x] \rrbracket=a^{*} \llbracket \operatorname{rec} x . t \rrbracket .
$$

Nondeterministic sum. Suppose $\Sigma_{i \in I} t_{i} \stackrel{a}{\rightarrow} t^{\prime}$ is derived from $t_{j} \stackrel{a}{\rightarrow} t^{\prime}$ for some $j \in I$. By the induction hypothesis and linearity of $a^{*}$,

$$
\llbracket ! t^{\prime} \rrbracket \subseteq a^{*} \llbracket t_{j} \rrbracket=\llbracket a^{*} t_{j} \rrbracket \subseteq \llbracket \Sigma_{i \in I} a^{*} t_{i} \rrbracket=a^{*} \llbracket \Sigma_{i \in I} t_{i} \rrbracket .
$$


Abstraction. Suppose $\lambda x . t \stackrel{u \mapsto a}{\longrightarrow} t^{\prime}$ is derived from $t[u / x] \stackrel{a}{\rightarrow} t^{\prime}$. By the induction hypothesis and Corollary 3.3,

$$
\llbracket ! t^{\prime} \rrbracket \subseteq a^{*} \llbracket t[u / x] \rrbracket=a^{*} \llbracket(\lambda x . t) u \rrbracket=(u \mapsto a)^{*} \llbracket \lambda x . t \rrbracket .
$$

Application. Suppose $t u \stackrel{a}{\rightarrow} t^{\prime}$ is derived from $t \stackrel{u \mapsto a}{\longrightarrow} t^{\prime}$. By the induction hypothesis,

$$
\llbracket ! t^{\prime} \rrbracket \subseteq(u \mapsto a)^{*} \llbracket t \rrbracket=a^{*} \llbracket t u \rrbracket .
$$

Injection. Suppose $\beta t \stackrel{\beta a}{\longrightarrow} t^{\prime}$ is derived from $t \stackrel{a}{\rightarrow} t^{\prime}$. By the induction hypothesis and Proposition 3.4,

$$
\llbracket ! t^{\prime} \rrbracket \subseteq a^{*} \llbracket t \rrbracket=a^{*} \llbracket \pi_{\beta}(\beta t) \rrbracket=(\beta a)^{*} \llbracket \beta t \rrbracket .
$$

Projection. Suppose $\pi_{\beta} t \stackrel{a}{\rightarrow} t^{\prime}$ is derived from $t \stackrel{\beta a}{\longrightarrow} t^{\prime}$. By the induction hypothesis,

$$
\llbracket ! t^{\prime} \rrbracket \subseteq(\beta a)^{*} \llbracket t \rrbracket=a^{*} \llbracket \pi_{\beta} t \rrbracket .
$$

Prefixing. Consider the transition $! t \stackrel{!}{\rightarrow} t$. By definition, $\llbracket ! t \rrbracket=!^{*} \llbracket ! t \rrbracket$.

Prefix match. Suppose $[u>! x \Rightarrow t] \stackrel{a}{\rightarrow} t^{\prime}$ is derived from $u \stackrel{!}{\rightarrow} u^{\prime}$ and $t\left[u^{\prime} / x\right] \stackrel{a}{\rightarrow} t^{\prime}$. By the induction hypothesis for $u$, we have $\llbracket ! u^{\prime} \rrbracket \subseteq !^{*} \llbracket u \rrbracket=\llbracket u \rrbracket$, and so by the induction hypothesis for $t$, Proposition 3.5, and monotonicity,

$$
\llbracket ! t^{\prime} \rrbracket \subseteq a^{*} \llbracket t\left[u^{\prime} / x\right] \rrbracket=a^{*} \llbracket\left[! u^{\prime}>! x \Rightarrow t\right] \rrbracket \subseteq a^{*} \llbracket[u>! x \Rightarrow t] \rrbracket .
$$

Fold. Suppose abs $t \stackrel{\text { abs } a}{\longrightarrow} t^{\prime}$ is derived from $t \stackrel{a}{\rightarrow} t^{\prime}$. By the induction hypothesis and Proposition 3.6,

$$
\llbracket ! t^{\prime} \rrbracket \subseteq a^{*} \llbracket t \rrbracket=a^{*} \llbracket \operatorname{rep}(a b s t) \rrbracket=(a b s a)^{*} \llbracket a b s t \rrbracket .
$$

Unfold. Suppose rept $\stackrel{a}{\rightarrow} t^{\prime}$ is derived from $t \stackrel{a b s a}{\longrightarrow} t^{\prime}$. By the induction hypothesis,

$$
\llbracket ! t^{\prime} \rrbracket \subseteq(\text { abs } a)^{*} \llbracket t \rrbracket=a^{*} \llbracket r e p t \rrbracket .
$$

The rule-induction is complete.

We obtain a corresponding adequacy result using logical relations $X \unlhd_{\mathbb{P}} t$ between subsets $X \subseteq \mathbb{P}$ and closed terms of type $\mathbb{P}$. Intuitively, $X \unlhd_{\mathbb{P}} t$ means that all paths in $X$ can be "operationally realised" by $t$. Because of recursive types, these relations cannot be defined by structural induction on the type $\mathbb{P}$ and we therefore employ a trick essentially due to Martin-Löf 
(see [24], Ch. 13). We define auxiliary relations $p \epsilon_{\mathbb{P}} t$ between paths $p: \mathbb{P}$ and closed terms $t$ of type $\mathbb{P}$, by induction on the structure of $p$ :

$$
\begin{aligned}
& X \unlhd_{\mathbb{P}} t \Longleftrightarrow \Longleftrightarrow_{\text {def }} \forall p \in X . p \epsilon_{\mathbb{P}} t \\
& P \mapsto q \epsilon_{\mathbb{P} \rightarrow \mathbb{Q}} t \Longleftrightarrow{ }_{\text {def }} \forall u .\left(P \unlhd_{\mathbb{P}} u \Longrightarrow q \epsilon_{\mathbb{Q}} t u\right) \\
& \beta p \epsilon_{\Sigma_{\alpha \in A} \mathbb{P}_{\alpha}} t \Longleftrightarrow \text { def } p \epsilon_{\mathbb{P}_{\beta}} \pi_{\beta} t \\
& P \epsilon_{! \mathbb{P}} t \Longleftrightarrow{ }_{\text {def }} \exists t^{\prime} . ! \mathbb{P}: t \stackrel{!}{\rightarrow} t^{\prime}: \mathbb{P} \text { and } P \unlhd_{\mathbb{P}} t^{\prime} \\
& \text { absp } \epsilon_{\mu_{j} \vec{T} \cdot \overrightarrow{\mathbb{T}}} t \Longleftrightarrow \text { def } p \epsilon_{\mathbb{T}_{j}[\mu \vec{T} \cdot \overrightarrow{\mathbb{T}} / \vec{T}]} \text { rept }
\end{aligned}
$$

Lemma 4.4 Suppose $\vdash t: \mathbb{P}$. Then $\llbracket t \rrbracket \unlhd_{\mathbb{P}} t$.

Proof. We need two technical results, which can both be proved by induction on the structure of paths. One says that $\epsilon_{\mathbb{P}}$ is closed on the left by $\leq_{\mathbb{P}}$, the

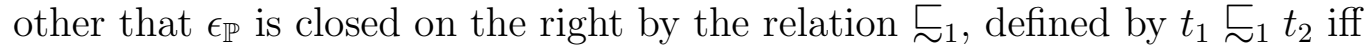
$\mathbb{P}: t_{1} \stackrel{a}{\rightarrow} t^{\prime}: \mathbb{P}^{\prime}$ implies $\mathbb{P}: t_{2} \stackrel{a}{\rightarrow} t^{\prime}: \mathbb{P}^{\prime}$.

Lemma 4.5 If $p \leq_{\mathbb{P}} p^{\prime}$ and $p^{\prime} \epsilon_{\mathbb{P}} t$, then $p \epsilon_{\mathbb{P}} t$.

Lemma 4.6 If $p \epsilon_{\mathbb{P}} t_{1}$ and $t_{1} \succsim_{1} t_{2}$, then $p \epsilon_{\mathbb{P}} t_{2}$.

It follows from Lemma 4.5 that for any subset $X$ of $\mathbb{P}$ we have $X \unlhd_{\mathbb{P}} t$ iff the down-closure of $X$, written $\bar{X}$, satisfies $\bar{X} \unlhd_{\mathbb{P}} t$. Lemma 4.6 will be used freely below.

The proof of the main lemma proceeds by structural induction on terms using the induction hypothesis

Suppose $x_{1}: \mathbb{P}_{1}, \ldots, x_{k}: \mathbb{P}_{k} \vdash t: \mathbb{P}$ and let $\vdash s_{j}: \mathbb{P}_{j}$ with $X_{j} \unlhd_{\mathbb{P}_{j}}$ $s_{j}$ for $1 \leq j \leq k$. Then $\llbracket t \rrbracket\left(\bar{X}_{1}, \ldots, \bar{X}_{k}\right) \unlhd_{\mathbb{P}} t\left[s_{1} / x_{1}, \ldots, s_{k} / x_{k}\right]$.

We'll abbreviate $x_{1}: \mathbb{P}_{1}, \ldots, x_{k}: \mathbb{P}_{k}$ to $\Gamma,\left(\bar{X}_{1}, \ldots, \bar{X}_{k}\right)$ to $X$, and the substitution $\left[s_{1} / x_{1}, \ldots, s_{k} / x_{k}\right]$ to $[s]$.

Variable. Let $\Gamma \vdash x_{j}: \mathbb{P}_{j}$, with $j$ between 1 and $k$, and $\vdash s_{j}: \mathbb{P}_{j}$ with $X_{j} \unlhd_{\mathbb{P}_{j}} s_{j}$ for $1 \leq j \leq k$. We must show that $\llbracket x_{j} \rrbracket X \unlhd_{\mathbb{P}_{j}} x_{j}[s]$. Now, $\llbracket x_{j} \rrbracket X=\bar{X}_{j}$ and $x_{j}[s] \equiv s_{j}$ so this amounts to $\bar{X}_{j} \unlhd_{\mathbb{P}_{j}} s_{j}$ which by the remarks above is equivalent to $X_{j} \unlhd_{\mathbb{P}_{j}} s_{j}$.

Recursive definition. Let $\Gamma \vdash$ rec x.t $: \mathbb{P}$ and $\vdash s_{j}: \mathbb{P}_{j}$ with $X_{j} \unlhd_{\mathbb{P}_{j}} s_{j}$ for $1 \leq j \leq k$. We must show that $\llbracket$ rec x.t $\rrbracket X \unlhd_{\mathbb{P}}$ rec x.t $[s]$. Now, $\llbracket$ rec x.t $\rrbracket X=$ $($ fix $f) X$ where $f$ maps $g: \Gamma \rightarrow \mathbb{P}$ to the composition

$$
\Gamma \stackrel{\Delta_{\Gamma}}{\longrightarrow} \Gamma \& \Gamma \stackrel{1_{\Gamma} \& g}{\longrightarrow} \Gamma \& \mathbb{P} \stackrel{t}{\rightarrow} \mathbb{P} .
$$


We'll show by induction on $n$ that $f^{n}(\varnothing) X \unlhd_{\mathbb{P}}$ rec $x . t[s]$ for all $n \in \omega$. Having done so we may argue as follows: Since

$$
\llbracket r e c x . t \rrbracket X=(f i x f) X=\left(\bigcup_{n \in \omega} f^{n} \varnothing\right) X=\bigcup_{n \in \omega}\left(\left(f^{n} \varnothing\right) X\right),
$$

we have that $p \in \llbracket$ rec $x . t \rrbracket X$ implies the existence of an $n \in \omega$ such that $p \in\left(f^{n} \varnothing\right) X$. Therefore $\llbracket$ rec $x . t \rrbracket X \unlhd_{\mathbb{P}}$ rec x.t $[s]$ as wanted.

Basis. Here, $\left(f^{0} \varnothing\right) X=\varnothing$. By definition of $\unlhd_{\mathbb{P}}$ we get $\varnothing \unlhd_{\mathbb{P}} t$ for any type $\mathbb{P}$ and term $\vdash t: \mathbb{P}$.

Step. Suppose $\left(f^{n} \varnothing\right) X \unlhd_{\mathbb{P}}$ rec x.t[s]. By the assumption of the lemma, $X_{j} \unlhd_{\mathbb{P}} s_{j}$ for each $1 \leq j \leq k$, and so by the induction hypothesis of the structural induction,

$$
\llbracket t \rrbracket\left(X,\left(f^{n} \varnothing\right) X\right) \unlhd_{\mathbb{P}} t[s][\text { rec } x . t[s] / x] .
$$

So if $p \in\left(f^{n+1} \varnothing\right) X$, then since $\left(f^{n+1} \varnothing\right) X=\llbracket t \rrbracket\left(X,\left(f^{n} \varnothing\right) X\right)$ we have $p \epsilon_{\mathbb{P}} t[s][$ rec x.t $[s] / x]$. By the transition rules we have $t[s][$ rec x.t $[s] / x] \succsim_{1}$ rec $x . t[s]$, and so $p \epsilon_{\mathbb{P}}$ rec x.t $[s]$. We conclude $\left(f^{n+1} \varnothing\right) X \unlhd_{\mathbb{P}}$ rec x.t $[s]$ and the mathematical induction is complete.

Nondeterministic sum. Let $\Gamma \vdash \Sigma_{i \in I} t_{i}: \mathbb{P}$ and $\vdash s_{j}: \mathbb{P}_{j}$ with $X_{j} \unlhd_{\mathbb{P}_{j}} s_{j}$ for $1 \leq j \leq k$. We must show that $\llbracket \Sigma_{i \in I} t_{i} \rrbracket X \unlhd_{\mathbb{P}} \Sigma_{i \in I} t_{i}[s]$. Now, $\llbracket \Sigma_{i \in I} t_{i} \rrbracket X=$ $\Sigma_{i \in I} \llbracket t_{i} \rrbracket X$. So if $p \in \llbracket \Sigma_{i \in I} t_{i} \rrbracket X$, there exists $j \in I$ with $p \in \llbracket t_{j} \rrbracket X$. Using the induction hypothesis for $t_{j}$ we have $p \epsilon_{\mathbb{P}} t_{j}[s]$. By the transition rules, $t_{j}[s] \sqsubseteq_{1} \Sigma_{i \in I} t_{i}[s]$ and so $p \epsilon_{\mathbb{P}} \Sigma_{i \in I} t_{i}[s]$ as wanted.

Abstraction. Let $\Gamma \vdash \lambda x . t: \mathbb{P} \rightarrow \mathbb{Q}$ and $\vdash s_{j}: \mathbb{P}_{j}$ with $X_{j} \unlhd_{\mathbb{P}_{j}} s_{j}$ for $1 \leq j \leq$ $k$. We must show that $\llbracket \lambda x . t \rrbracket X \unlhd_{\mathbb{P} \rightarrow \mathbb{Q}}(\lambda x . t)[s]$. So let $P \mapsto q \in \llbracket \lambda x . t \rrbracket X$. By the denotational semantics, we then have $q \in \llbracket t \rrbracket\left(X, i_{\mathbb{P}} P\right)$. We must show that $P \mapsto q \epsilon_{\mathbb{P} \rightarrow \mathbb{Q}}(\lambda x . t)[s]$. So suppose $\vdash u: \mathbb{P}$ with $P \unlhd_{\mathbb{P}} u$. We must then show $q \epsilon_{\mathbb{Q}}(\lambda x . t)[s] u$. By the transition rules, $t[s][u / x] \sqsubseteq_{1}(\lambda x . t)[s] u$ and so it is sufficient to show $q \epsilon_{\mathbb{Q}} t[s][u / x]$. Now, by the induction hypothesis, we know that $\llbracket t \rrbracket\left(X, i_{\mathbb{P}} P\right) \unlhd_{\mathbb{Q}} t[s][u / x]$ and so, with $q \in \llbracket t \rrbracket\left(X, i_{\mathbb{P}} P\right)$, we are done.

Application. Let $\Gamma \vdash t u: \mathbb{Q}$ and $\vdash s_{j}: \mathbb{P}_{j}$ with $X_{j} \unlhd_{\mathbb{P}_{j}} s_{j}$ for $1 \leq j \leq k$. We must show that $\llbracket t u \rrbracket X \unlhd_{\mathbb{Q}}(t u)[s]$. So suppose $q \in \llbracket t u \rrbracket X$. By the denotational semantics, there exists $P \in ! \mathbb{P}$ such that $P \mapsto q \in \llbracket t \rrbracket X$ and $P \subseteq \llbracket u \rrbracket X$. By the induction hypothesis for $t$, we have $\llbracket t \rrbracket X \unlhd_{\mathbb{P} \rightarrow \mathbb{Q}} t[s]$ and so $P \mapsto q \epsilon_{\mathbb{P} \rightarrow \mathbb{Q}} t[s]$. This means that given any $\vdash u^{\prime}: \mathbb{P}$ with $P \unlhd_{\mathbb{P}} u^{\prime}$, we have $q \epsilon_{\mathbb{Q}} t[s] u^{\prime}$. Now using the induction hypothesis for $u$ we get that $\llbracket u \rrbracket X \unlhd_{\mathbb{P}} u[s]$ and so, since $P \subseteq \llbracket u \rrbracket X$, we have $P \unlhd_{\mathbb{P}} u[s]$ so that $q \epsilon_{\mathbb{Q}} t[s] u[s] \equiv(t u)[s]$ as wanted. 
Injection. Let $\Gamma \vdash \beta t: \Sigma_{\alpha \in A} \mathbb{P}_{\alpha}$ and $\vdash s_{j}: \mathbb{P}_{j}$ with $X_{j} \unlhd_{\mathbb{P}_{j}} s_{j}$ for $1 \leq j \leq k$. We must show that $\llbracket \beta t \rrbracket X \unlhd_{\Sigma_{\alpha \in A} \mathbb{P}_{\alpha}}(\beta t)[s]$. So suppose $\beta p \in \llbracket \beta t \rrbracket X$; by the denotational semantics, $p \in \llbracket t \rrbracket X$. We must then show that $\beta p \epsilon_{\Sigma_{\alpha \in A} \mathbb{P}_{\alpha}}$ $(\beta t)[s]$ which means that $p \epsilon_{\mathbb{P}_{\beta}} \pi_{\beta}(\beta t[s])$. By the transition rules, we have $t[s] \succsim_{1} \pi_{\beta}(\beta t[s])$ so it is sufficient to show that $p \in_{\mathbb{P}_{\beta}} t[s]$. By the induction hypothesis, $\llbracket t \rrbracket X \unlhd_{\mathbb{P}_{\beta}} t[s]$ and so, since $p \in \llbracket t \rrbracket X$ we have $p \epsilon_{\mathbb{P}_{\beta}} t[s]$ as wanted.

Projection. Let $\Gamma \vdash \pi_{\beta} t: \mathbb{P}_{\beta}$ with $\Gamma \vdash t: \Sigma_{\alpha \in A} \mathbb{P}_{\alpha}$ and $\beta \in A$, and $\vdash s_{j}: \mathbb{P}_{j}$ with $X_{j} \unlhd_{\mathbb{P}_{j}} s_{j}$ for $1 \leq j \leq k$. We must show that $\llbracket \pi_{\beta} t \rrbracket X \unlhd_{\mathbb{P}_{\beta}} \pi_{\beta} t[s]$. So suppose $p \in \llbracket \pi_{\beta} t \rrbracket X$; by the denotational semantics, $\beta p \in \llbracket t \rrbracket X$. By the induction hypothesis, $\llbracket t \rrbracket X \unlhd_{\Sigma_{\alpha \in A} \mathbb{P}_{\alpha}} t[s]$ and so $\beta p \epsilon_{\Sigma_{\alpha \in A} \mathbb{P}_{\alpha}} t[s]$ which means that $p \epsilon_{\mathbb{P}_{\beta}} \pi_{\beta} t[s]$ as wanted.

Prefixing. Let $\Gamma \vdash ! t: \mathbb{P}$ and $\vdash s_{j}: \mathbb{P}_{j}$ with $X_{j} \unlhd_{\mathbb{P}_{j}} s_{j}$ for $1 \leq j \leq k$. We must show that $\llbracket ! t \rrbracket X \unlhd ! \mathbb{P} ! t[s]$. So suppose $P \in \llbracket ! t \rrbracket X$; by the denotational semantics, $P \subseteq \llbracket t \rrbracket X$. We must then show that $P \epsilon_{! \mathbb{P}} ! t[s]$, and so since the transition rules provide a derivation $! \mathbb{P}: ! t[s] \stackrel{!}{\rightarrow} t[s]: \mathbb{P}$, it is enough to show that $P \unlhd_{\mathbb{P}} t[s]$. Now, by the induction hypothesis, $\llbracket t \rrbracket X \unlhd_{\mathbb{P}} t[s]$ and so, since $P \subseteq \llbracket t \rrbracket X$ we have $P \unlhd_{\mathbb{P}} t[s]$ as wanted.

Prefix match. Let $\Gamma \vdash[u>! x \Rightarrow t]: \mathbb{Q}$ and $\vdash s_{j}: \mathbb{P}_{j}$ with $X_{j} \unlhd_{\mathbb{P}_{j}} s_{j}$ for $1 \leq j \leq k$. By renaming $x$ if necessary, we may assume that $x$ is not one of the $x_{j}$. We must show that $\llbracket[u>! x \Rightarrow t] \rrbracket X \unlhd_{\mathbb{Q}}[u>! x \Rightarrow t][s]$. So suppose $q \in \llbracket[u>! x \Rightarrow t] \rrbracket X$; by the denotational semantics, there exists $P \in ! \mathbb{P}$ such that $q \in \llbracket t \rrbracket\left(X, i_{\mathbb{P}} P\right)$ and $P \in \llbracket u \rrbracket X$. By the induction hypothesis for $u$ we have $\llbracket u \rrbracket X \unlhd_{! \mathbb{P}} u[s]$ and so since $P \in \llbracket u \rrbracket X$, there exists $u^{\prime}$ such that $! \mathbb{P}: u[s] \stackrel{!}{\rightarrow} u^{\prime}: \mathbb{P}$ and $P \unlhd_{\mathbb{P}} u^{\prime}$. Hence, by the induction hypothesis for $t$ we have $\llbracket t \rrbracket\left(X, i_{\mathbb{P}} P\right) \unlhd_{\mathbb{Q}} t[s]\left[u^{\prime} / x\right]$ and so since $q \in \llbracket t \rrbracket\left(X, i_{\mathbb{P}} P\right)$ we have $q \epsilon_{\mathbb{Q}} t[s]\left[u^{\prime} / x\right]$. Now, by the transition rules, $t[s]\left[u^{\prime} / x\right] \succsim_{1}[u>! x \Rightarrow t][s]$ and so $q \epsilon_{\mathbb{Q}}[u>! x \Rightarrow t][s]$ as wanted.

Fold. Let $\Gamma \vdash$ abst $: \mu_{j} \vec{T} \cdot \overrightarrow{\mathbb{T}}$ and $\vdash s_{j}: \mathbb{P}_{j}$ with $X_{j} \unlhd_{\mathbb{P}_{j}} s_{j}$ for $1 \leq j \leq k$. We must show that $\llbracket a b s t \rrbracket X \unlhd_{\mu_{j} \vec{P} . \overrightarrow{\mathbb{T}}}$ abs $t[s]$. So suppose absq $\in \llbracket a b s t \rrbracket X$ such that $q \in \llbracket t \rrbracket X$. By the induction hypothesis, $q \epsilon_{\mathbb{T}_{j}[\mu \vec{T} \cdot \overrightarrow{\mathbb{T}} / \vec{T}]} t[s]$ and since $t[s] \complement_{1}$ rep abst $\left.t s\right]$, we have $q \epsilon_{\mathbb{T}_{j}[\mu \vec{T} \cdot \overrightarrow{\mathbb{T}} / \vec{T}]}$ rep abs $t[s]$ which means that abs $q \epsilon_{\mu_{j} \vec{P} . \overrightarrow{\mathbb{T}}}$ abst $[s]$ as wanted.

Unfold. Let $\Gamma \vdash \operatorname{rep} t: \mathbb{T}_{j}[\mu \vec{T} \cdot \overrightarrow{\mathbb{T}} / \vec{T}]$ and $\vdash s_{j}: \mathbb{P}_{j}$ with $X_{j} \unlhd_{\mathbb{P}_{j}} s_{j}$ for $1 \leq j \leq$ $k$. We must show that $\llbracket$ rep $t \rrbracket X \unlhd_{\mathbb{T}_{j}[\mu \vec{T} . \overrightarrow{\mathbb{T}} / \vec{T}]} \operatorname{rep} t[s]$. So suppose $q \in \llbracket$ rep $t \rrbracket X$ such that abs $q \in \llbracket t \rrbracket X$. By the induction hypothesis, abs $q \epsilon_{\mu_{j} \vec{T} . \overrightarrow{\mathbb{T}}} t[s]$ and so $q \epsilon_{\mathbb{T}_{j}[\mu \vec{T} \cdot \overrightarrow{\mathbb{T}} / \vec{T}]}$ rept $[s]$ as wanted.

The structural induction is complete. 
Proposition 4.7 (Adequacy) Suppose $\vdash t: \mathbb{P}$ and $\mathbb{P}: a: \mathbb{P}^{\prime}$. Then

$$
a^{*} \llbracket t \rrbracket \neq \varnothing \Longleftrightarrow \exists t^{\prime} . \mathbb{P}: t \stackrel{a}{\rightarrow} t^{\prime}: \mathbb{P}^{\prime}
$$

Proof. The " $\Leftarrow$ " direction follows from soundness. Assume $a^{*} \llbracket t \rrbracket \neq \varnothing$. Then because $a^{*} \llbracket t \rrbracket$ is a downwards-closed subset of $! \mathbb{P}^{\prime}$ which has least element $\varnothing$, we must have $\varnothing \in a^{*} \llbracket t \rrbracket$. Thus $\varnothing{\epsilon ! \mathbb{\mathbb { P } ^ { \prime }}} a^{*} t$ by Lemma 4.4 , which implies the existence of a term $t^{\prime}$ such that $! \mathbb{P}^{\prime}: a^{*} t \stackrel{!}{\rightarrow} t^{\prime}: \mathbb{P}^{\prime}$. By Lemma 4.2 we have $\mathbb{P}: t \stackrel{a}{\rightarrow} t^{\prime}: \mathbb{P}^{\prime}$.

\subsection{Full Abstraction w.r.t. Operational Semantics}

Adequacy allows an operational formulation of contextual equivalence. If $t$ is a program, we write $t \stackrel{!}{\rightarrow}$ if there exists $t^{\prime}$ such that $! \mathbb{O}: t \stackrel{!}{\rightarrow} t^{\prime}: \mathbb{O}$. We then have $t \stackrel{!}{\rightarrow}$ iff $\llbracket t \rrbracket \neq \varnothing$ by adequacy. Hence, two terms $t_{1}$ and $t_{2}$ with $\Gamma \vdash t_{1}: \mathbb{P}$ and $\Gamma \vdash t_{2}: \mathbb{P}$ are related by contextual preorder iff for all $(\Gamma, \mathbb{P})$-program contexts $C$, we have $C\left(t_{1}\right) \stackrel{!}{\rightarrow} \Longrightarrow C\left(t_{2}\right) \stackrel{!}{\rightarrow}$.

Full abstraction is often formulated in terms of this operational preorder. With $t_{1}$ and $t_{2}$ as above, the inclusion $\llbracket t_{1} \rrbracket \subseteq \llbracket t_{2} \rrbracket$, holds iff for all $(\Gamma, \mathbb{P})$ program contexts $C$, we have the implication $C\left(t_{1}\right) \stackrel{!}{\rightarrow} \Longrightarrow C\left(t_{2}\right) \stackrel{!}{\rightarrow}$.

\subsection{Simulation}

The path semantics does not capture enough of the branching behaviour of processes to characterise bisimilarity (for that, the presheaf semantics is needed, see $[11,19])$. As an example, the processes $! \varnothing+! ! \varnothing$ and $! ! \varnothing$ have the same denotation, but are clearly not bisimilar. However, using Hennessy-Milner logic we can link path equivalence to simulation. In detail, we consider the fragment of Hennessy-Milner logic given by possibility and finite conjunctions; it is characteristic for simulation equivalence in the case of image-finite processes [8]. With $a$ ranging over actions, formulae are given by

$$
\phi::=\langle a\rangle \phi \mid \bigwedge_{i \leq n} \phi_{i} .
$$

The empty conjunction is written $T$. We type formulae using judgements $\phi: \mathbb{P}$, the idea being that only processes of type $\mathbb{P}$ should be described by $\phi: \mathbb{P}$.

$$
\frac{\mathbb{P}: a: \mathbb{P}^{\prime} \quad \phi: \mathbb{P}^{\prime}}{\langle a\rangle \phi: \mathbb{P}} \quad \frac{\phi_{i}: \mathbb{P} \text { all } i \leq n}{\bigwedge_{i \leq n} \phi_{i}: \mathbb{P}}
$$


The notion of satisfaction, written $t \vDash \phi: \mathbb{P}$, is defined by

$$
\begin{aligned}
t \vDash\langle a\rangle \phi: \mathbb{P} & \Longleftrightarrow \exists t^{\prime} . \mathbb{P}: t \stackrel{a}{\rightarrow} t^{\prime}: \mathbb{P}^{\prime} \text { and } t^{\prime} \vDash \phi: \mathbb{P}^{\prime} \\
t \vDash \bigwedge_{i \leq n} \phi_{i}: \mathbb{P} & \Longleftrightarrow t \vDash \phi_{i}: \mathbb{P} \text { for each } i \leq n
\end{aligned}
$$

Note that $\top: \mathbb{P}$ and $t \vDash \top: \mathbb{P}$ for all $\vdash t: \mathbb{P}$.

Definition 4.8 Closed terms $t_{1}, t_{2}$ of the same type $\mathbb{P}$ are related by logical preorder, written $t_{1} \varlimsup_{\mathrm{L}} t_{2}$, iff for all formulae $\phi: \mathbb{P}$ we have $t_{1} \vDash \phi: \mathbb{P} \Longrightarrow$ $t_{2} \vDash \phi: \mathbb{P}$. If both $t_{1} \succsim_{\mathrm{L}} t_{2}$ and $t_{2} \succsim_{\mathrm{L}} t_{1}$, we say that $t_{1}$ and $t_{2}$ are logically equivalent.

Using adequacy and by adapting the proof of full abstraction, we can show that logical equivalence coincides with contextual equivalence as do the associated preorders:

Theorem 4.9 For closed terms $t_{1}$ and $t_{2}$ of the same type $\mathbb{P}$,

$$
t_{1} \sqsubseteq t_{2} \Longleftrightarrow t_{1} \check{L}_{\mathrm{L}} t_{2}
$$

Proof. To each formula $\phi: \mathbb{P}$ we can construct a $(\mathbb{O}, \mathbb{P})$-program context $C_{\phi}$ with the property that

$$
\text { !O }: C_{\phi}(t) \stackrel{!}{\rightarrow} \Longleftrightarrow t \vDash \phi: \mathbb{P}
$$

Define

$$
\begin{gathered}
C_{\langle u \mapsto a\rangle \phi} \equiv_{\text {def }} C_{\langle a\rangle \phi}(-u), \quad C_{\langle!\rangle \phi} \equiv_{\text {def }}\left[->! x \Rightarrow C_{\phi}(x)\right], \\
C_{\langle\beta a\rangle \phi} \equiv_{\text {def }} C_{\langle a\rangle \phi}\left(\pi_{\beta}-\right), \quad C_{\langle a b s a\rangle \phi} \equiv_{\text {def }} C_{\langle a\rangle \phi}(\text { rep }-), \\
C_{\bigwedge_{i \leq n} \phi_{i}} \equiv_{\text {def }}\left[C_{\phi_{1}}>! x_{1} \Rightarrow \cdots \Rightarrow\left[C_{\phi_{n}}>! x_{n} \Rightarrow ! \varnothing\right] \cdots\right] .
\end{gathered}
$$

It follows by $(65)$ that $t_{1} \varlimsup_{\mathrm{L}} t_{2}$ iff for all formulae $\phi: \mathbb{P}$ we have that $C_{\phi}\left(t_{1}\right) \stackrel{!}{\rightarrow}$ implies $C_{\phi}\left(t_{2}\right) \stackrel{!}{\rightarrow}$. The direction " $\Rightarrow$ " then follows by adequacy.

For the converse, we observe that the program contexts $C_{p}$ used in the full-abstraction proof are all subsumed by the contexts $C_{\phi}$. In detail, using the terms $t_{P}^{\prime}$ realising finite sets of paths, we can define actions $\mathbb{P}: a_{p}: \mathbb{P}^{\prime}$ and formulae $\phi_{p}: \mathbb{P}$ by induction on paths $p: \mathbb{P}$ such that $C_{p} \equiv C_{\left\langle a_{p}\right\rangle \phi_{p}}$ :

$$
\begin{aligned}
a_{P \mapsto q} & \equiv_{\text {def }} t_{P}^{\prime} \mapsto a_{q} & \phi_{P \mapsto q} & \equiv_{\text {def }} \phi_{q} \\
a_{\beta p} & \equiv_{\text {def }} \beta a_{p} & \phi_{\beta p} & \equiv_{\text {def }} \phi_{p} \\
a_{P} & \equiv_{\text {def }} ! & \phi_{P} & \equiv_{\text {def }} \bigwedge_{p \in P}\left\langle a_{p}\right\rangle \phi_{p} \\
a_{a b s p} & \equiv_{\text {def }} \text { abs } a_{p} & \phi_{a b s p} & \equiv_{\text {def }} \phi_{p}
\end{aligned}
$$


With $p: \mathbb{P}$ and $\vdash t: \mathbb{P}$ we obtain $p \in \llbracket t \rrbracket$ iff $\llbracket C_{\left\langle a_{p}\right\rangle \phi_{p}}(t) \rrbracket \neq \varnothing$ as in the proof of full abstraction, and so by adequacy and (65), we have $p \in \llbracket t \rrbracket$ iff $t \vDash\left\langle a_{p}\right\rangle \phi_{p}: \mathbb{P}$. It follows that $t_{1} \varlimsup_{\mathrm{L}} t_{2}$ implies $\llbracket t_{1} \rrbracket \subseteq \llbracket t_{2} \rrbracket$, and so $t_{1} \sqsubseteq t_{2}$.

We note that the proof above establishes a link between paths and actions:

$$
p \in \llbracket t \rrbracket \Longleftrightarrow \mathbb{P}: t \stackrel{a_{p}}{\longrightarrow} t^{\prime}: \mathbb{P}^{\prime} \text { and } t^{\prime} \vDash \phi_{p}: \mathbb{P}^{\prime}
$$

\section{$5 \quad$ Related and Future Work}

Matthew Hennessy's fully abstract semantics for higher-order CCS [9] is a path semantics, and what we have presented here can be seen as a generalisation of his work via the translation of higher-order CCS into HOPLA, see [19].

The presheaf semantics originally given for HOPLA is a refined version of the path semantics. A path set $X \in \widehat{\mathbb{P}}$ can be seen to give a "yes/no answer" to the question of whether or not a path $p \in \mathbb{P}$ can be realised by the process (cf. the representation in Sect. 2 of path sets as monotone maps $\mathbb{P}^{\text {op }} \rightarrow 2$ ). A presheaf over $\mathbb{P}$ is a functor $\mathbb{P}^{\text {op }} \rightarrow$ Set to the category of sets and functions, and gives instead a set of "realisers", saying how a path may be realised. This extra information can be used to obtain refined versions of the proofs of soundness and adequacy, giving hope of extending the full abstraction result to a characterisation of bisimilarity, possibly in terms of open maps [11].

Replacing the exponential ! by a "lifting" comonad yields a model Aff of affine linear logic and an affine version of HOPLA, again with a fully abstract path semantics [20]. The tensor operation of Aff can be understood as a simple parallel composition of event structures [21]. Thus, the affine language holds promise of extending our approach to "independence" models like Petri nets or event structures in which computation paths are partial orders of events. Work is in progress to provide an operational semantics for this language together with results similar to those obtained here [21].

Being a higher-order process language, HOPLA allows process passing and so can express certain forms of mobility, in particular that present in the ambient calculus with public names $[3,19]$. Another kind of mobility, mobility of communication links, arises from name-generation as in the $\pi$ calculus [15]. Inspired by HOPLA, Francesco Zappa Nardelli and GW have defined a higher-order process language with name-generation, allowing encodings of full ambient calculus and $\pi$-calculus. Bisimulation properties and semantic underpinnings are being developed [26]. 


\section{References}

[1] P. N. Benton. A mixed linear and non-linear logic: proofs, terms and models (extended abstract). In Proc. CSL'94, LNCS 933.

[2] T. Bräuner. An Axiomatic Approach to Adequacy. Ph.D. dissertation, University of Aarhus, 1996. BRICS Dissertation Series DS-96-4.

[3] L. Cardelli and A. D. Gordon. Anytime, anywhere: modal logics for mobile ambients. In Proc. POPL'00.

[4] G. L. Cattani and G. Winskel. Profunctors, open maps and bisimulation. Manuscript, 2000.

[5] J.-Y. Girard. Linear logic. Theoretical Computer Science, 50(1):1-102, 1987.

[6] A. D. Gordon. Bisimilarity as a theory of functional programming. In Proc. MFPS'95, ENTCS 1.

[7] M. C. B. Hennessy and G. D. Plotkin. Full abstraction for a simple parallel programming language. In Proc. MFCS'ry, LNCS 74.

[8] M. Hennessy and R. Milner. Algebraic laws for nondeterminism and concurrency. Journal of the ACM, 32(1):137-161, 1985.

[9] M. Hennessy. A fully abstract denotational model for higher-order processes. Information and Computation, 112(1):55-95, 1994.

[10] C. A. R. Hoare. A Model for Communicating Sequential Processes. Technical monograph, PRG-22, University of Oxford Computing Laboratory, 1981.

[11] A. Joyal, M. Nielsen, and G. Winskel. Bisimulation from open maps. Information and Computation, 127:164-185, 1996.

[12] K. G. Larsen and G. Winskel. Using information systems to solve recursive domain equations effectively. In Proc. Semantics of Data Types, 1984, LNCS 173.

[13] G. McCusker. A fully abstract relational model of syntactic control of interference. In Proc. CSL'02, LNCS 2471.

[14] P.-A. Melliès. Categorical models of linear logic revisited. Submitted to Theoretical Computer Science, 2002.

[15] R. Milner, J. Parrow and D. Walker. A calculus of mobile processes, parts I and II. Information and Computation, 100(1):1-77, 1992. 
[16] J. H. Morris. Lambda-Calculus Models of Programming Languages. PhD thesis, MIT, 1968.

[17] M. Nielsen, G. Plotkin and G. Winskel. Petri nets, event structures and domains, part I. Theoretical Computer Science, 13(1):85-108, 1981.

[18] M. Nygaard and G. Winskel. Linearity in process languages. In Proc. LICS'02.

[19] M. Nygaard and G. Winskel. HOPLA - a higher-order process language. In Proc. CONCUR'02, LNCS 2421.

[20] M. Nygaard and G. Winskel. Domain theory for concurrency. Submitted to Theoretical Computer Science, 2003.

[21] M. Nygaard. Domain Theory for Concurrency. PhD dissertation, University of Aarhus, 2003.

[22] D. S. Scott. Domains for denotational semantics. In Proc. ICALP'82, LNCS 140.

[23] R. A. G. Seely. Linear logic, *-autonomous categories and cofree coalgebras. In Proc. Categories in Computer Science and Logic, 1987.

[24] G. Winskel. The Formal Semantics of Programming Languages. MIT Press, 1993.

[25] G. Winskel. A presheaf semantics of value-passing processes. In Proc. CONCUR'96, LNCS 1119.

[26] G. Winskel and F. Zappa Nardelli. Manuscript, 2003. 


\section{Recent BRICS Report Series Publications}

RS-03-42 Mikkel Nygaard and Glynn Winskel. Full Abstraction for HOPLA. December 2003. 25 pp. Appears in Amadio and Lugiez, editors, Concurrency Theory: 14th International Conference, CONCUR '03 Proceedings, LNCS 2761, 2003, pages 383-398.

RS-03-41 Malgorzata Biernacka, Dariusz Biernacki, and Olivier Danvy. An Operational Foundation for Delimited Continuations. December 2003. 21 pp.

RS-03-40 Andrzej Filinski and Henning Korsholm Rohde. A Denotational Account of Untyped Normalization by Evaluation. December 2003. 29 pp.

RS-03-39 Jörg Abendroth. Applying $\pi$-Calculus to Practice: An Example of a Unified Security Mechanism. November 2003. 35 pp.

RS-03-38 Henning Böttger, Anders Møller, and Michael I. Schwartzbach. Contracts for Cooperation between Web Service Programmers and HTML Designers. November 2003. 23 pp.

RS-03-37 Claude Crépeau, Paul Dumais, Dominic Mayers, and Louis Salvail. Computational Collapse of Quantum State with Application to Oblivious Transfer. November 2003. 30 pp.

RS-03-36 Ivan B. Damgård, Serge Fehr, Kirill Morozov, and Louis Salvail. Unfair Noisy Channels and Oblivious Transfer. November 2003.

RS-03-35 Mads Sig Ager, Olivier Danvy, and Jan Midtgaard. A Functional Correspondence between Monadic Evaluators and Abstract Machines for Languages with Computational Effects. November 2003. 31 pp.

RS-03-34 Luca Aceto, Willem Jan Fokkink, Anna Ingólfsdóttir, and Bas Luttik. CCS with Hennessy's Merge has no Finite Equational Axiomatization. November 2003. 37 pp.

RS-03-33 Olivier Danvy. A Rational Deconstruction of Landin's SECD Machine. October 2003. $32 \mathrm{pp}$. This report supersedes the earlier BRICS report RS-02-53.

RS-03-32 Philipp Gerhardy and Ulrich Kohlenbach. Extracting Herbrand Disjunctions by Functional Interpretation. October 2003. 17 pp. 